PHYSICAL REVIEW D 94, 054006 (2016)

\title{
Mapping chiral symmetry breaking in the excited baryon spectrum
}

\author{
Pedro Bicudo and Marco Cardoso \\ CFTP, Departamento de Física, Instituto Superior Técnico, \\ Avenida Rovisco Pais 1049-001 Lisboa, Portugal \\ Felipe J. Llanes-Estrada and Tim Van Cauteren \\ Departamento de Física Teórica I, Universidad Complutense de Madrid, 28040 Madrid, Spain
}

(Received 17 May 2016; published 7 September 2016)

\begin{abstract}
We study the conjectured "insensitivity to chiral symmetry breaking" in the highly excited light baryon spectrum. While the experimental spectrum is being measured at JLab and CBELSA/TAPS, this insensitivity remains to be computed theoretically in detail. As the only existing option to have both confinement, highly excited states, and chiral symmetry, we adopt the truncated Coulomb-gauge formulation of QCD, considering a linearly confining Coulomb term. Adopting a systematic and numerically intensive variational treatment up to 12 harmonic oscillator shells we are able to access several angular and radial excitations. We compute both the excited spectra of $I=1 / 2$ and $I=3 / 2$ baryons, up to large spin $J=13 / 2$, and study in detail the proposed chiral multiplets. While the static-light and light-light spectra clearly show chiral symmetry restoration high in the spectrum, the realization of chiral symmetry is more complicated in the baryon spectrum than earlier expected.
\end{abstract}

DOI: 10.1103/PhysRevD.94.054006

\section{INTRODUCTION}

In a short article [1] and several communications [2-6] we proposed a variational approach to the truncated Coulombgauge formulation of QCD, considering a linearly confining term, to gain novel insights into the baryon spectrum, particularly into the standing conjecture of insensitivity to chiral symmetry breaking in the high spectrum.

After our first result computed with Monte Carlo integration [1], we continued to refine our numerical technique, and opted for Gauss integration, in order to analyze with sufficient detail the splittings in the proposed chiral multiplets. We now provide full details of this research line and comprehensively report our methods and findings.

The fundamental motivation to embark on this investigation of possible chiral multiplets in the baryon spectrum is driven by works initiated by de Tar, Kunihiro, Cohen, and Glozman [7-17] in which a chiral degeneracy, or chiral symmetry restoration, was suggested to occur in the excited baryon spectrum. Detailed examination of the arguments therein requires addressing the excited spectrum up to several excitations while breaking chiral symmetry in a controlled manner.

We have found particularly interesting [1] the study of Yrast baryons (which, with the same usage of the Swedish word for "dizziest" as in nuclear physics, are the lowest mass baryons for each angular momentum $J$ ) as they can unambiguously be identified for each parity. This is an advantage over the case of radial-like excitations: there it is less clear which state of positive parity should be compared to which state of negative parity.

So far, large angular excitations have not been investigated in lattice gauge field-theory studies, and thus model studies are in order. The model computations for simpler hadron spectra, such as light-light mesons and static-light mesons, already theoretically support the concept [18-22]. The agreement [23] is that, in addition to parity doubling brought about by the symmetry, the pion couplings to various $N^{*}, \Delta^{*}$, etc. resonances become decreasingly small with the excitation quantum number (due to smaller wave function overlaps between the boosted decay-pion and incoming and outgoing $N^{*}$ 's).

In this work we employ the only model for baryons that can presently test the concept. In essence, one needs a confining model where high excitations, e.g., angular, can be studied. At the same time, we need a model where chiral symmetry is spontaneously, not explicitly, broken. As the only existing option to have confinement, highly excited states, and chiral symmetry, we adopt the truncated Coulomb-gauge formulation of QCD. Moreover, we consider the case of a linearly confining Coulomb term, necessary for linear Regge trajectories. The model Hamiltonian is given in Eq. (4.1) below, but suffice it to say here that the interaction vertex is chiral symmetric, entailing the spinor combination $U^{\dagger} U$ that corresponds to the nonrelativistic reduction of the chiral symmetry preserving, QED-like vertex $\bar{\psi} \gamma^{\mu} \psi$. We compute the mass spectra of positive parity and negative parity excited baryons, $M_{J}^{+}$and $M_{J}^{-}$, for both isospin $I=1 / 2$ and $I=3 / 2$, and compare them, looking for signs of chiral symmetry restoration. The parameters of interest are the differences between these four energies.

The truncated Coulomb-gauge approach is equivalent to a field-theory version of chiral invariant quark models [24-34]. Because the model includes linear confinement 


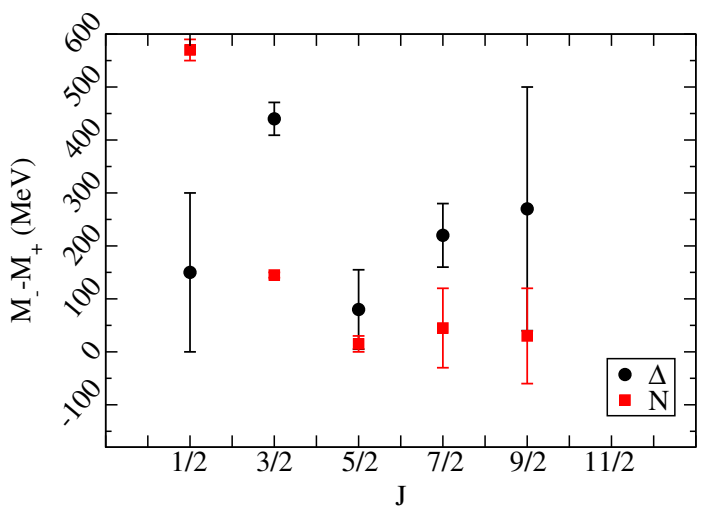

FIG. 1. Experimental parity splittings in the confirmed $N$ and $\Delta$ Yrast baryons as a function of angular momentum [54].

one can examine an excited spectrum with infinitely many excitations. We do not necessarily claim they should be found in experiment, as we do not treat open decay channels [35] in this work, given the already very large task at hand. Second, because of the field-theory formulation, one can employ the renowned BCS approximation to spontaneously break chiral symmetry by a ${ }^{3} P_{0}$ Cooperpair condensate [29-31] and thus control the sensitivity of the high spectrum to this breaking thanks to the dynamical quark mass.

As additional motivation, recent efforts at CBELSA/TAPS [36-38], CLAS, and CLAS12 at Jefferson Lab [39-41] and by the Bonn-Gatchina partial wave analysis group [42-45] show good prospects for progress in the excited baryon spectrum. Some of the resonances that we address, particularly for high angular momentum, are of experimental interest.

Surprisingly, the spectrum of excited baryons, well addressed within massive-quark models [46-51], remains to be studied in depth with a chirally invariant and linearly confining quark model, although many studies have followed the approach for the vacuum and mesons $[19,20,52,53]$. Here we provide the first detailed study of the baryon spectrum with such a chirally invariant approach and a linearly rising potential.

Before we report our computations, it is worth recalling the current experimental situation [54] in what concerns Yrast-baryon parity splittings as function of angular momentum. Figure 1 shows the difference of $N^{+}$and $N^{-}$masses (or parity splitting) as well as that of $\Delta^{+}$and $\Delta^{-}$ as a function of $J$. It is apparent from this figure that the nucleon splittings do seem smaller on average for larger $J$ consistently with the theory prediction, while the $\Delta$ splittings do not show clear evidence of decrease; they are at best constant, and the experimental uncertainty is large.

We also render, in Fig. 2, an estimate of the splittings that we have assembled from the lattice gauge theory calculation of the baryon spectrum reported in [55]. One can argue about whether the standing lattice data can be used to

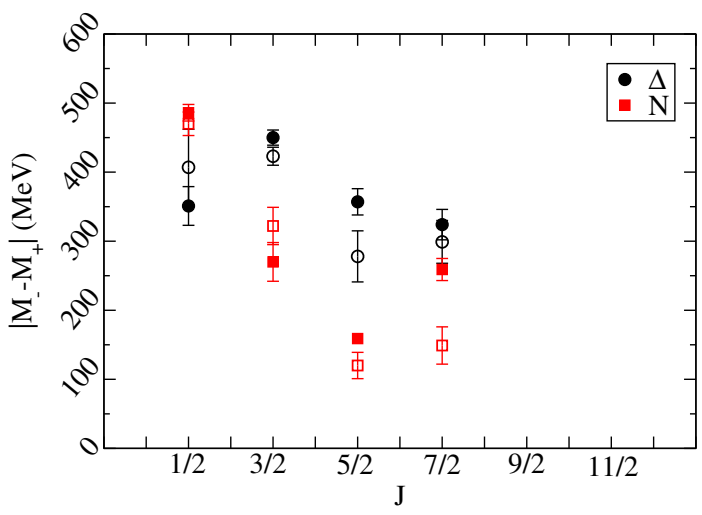

FIG. 2. Known parity splittings for $N$ and $\Delta$ ground states and angular excitations in QCD (our estimate from the lattice gauge theory computations reported in [55]). Solid symbols: $m_{\pi}=524 \mathrm{MeV}$. Hollow symbols: $m_{\pi}=391 \mathrm{MeV}$.

make a statement about spontaneous chiral symmetry breaking and its effect in the high spectrum, as the pion masses used in that reference are 524 and $391 \mathrm{MeV}$, respectively; chiral symmetry is explicitly broken. With much precaution, we may observe that there is some decrease in the parity splittings of the $J=5 / 2$ and $J=$ $7 / 2$ angular momentum channels in Fig. 2, but further calculations seem necessary.

From this brief inspection of the experimental and lattice data, there is clearly room for model insight if we can extend the calculation to large angular momenta (or other types of excitation). But meaningful models of this relativistic three-body system are not exactly solvable and require, as lattice gauge theory does, a massive computational effort, which we here report.

Unlike recent nuclear computations that also use the variational principle extensively to address the few-body problem [56,57], the major difficulty that we face here is not the diagonalization of huge matrices, but rather the computation of the matrix elements of the Hamiltonian. As is shown below in Sec. V, there is a lot of bookkeeping in the construction of the basis, but not massive computer time. Each matrix element, however, is a complicated integral that, without the simplifications brought about by the nonrelativistic limit (which, while being adequate in nuclear physics, are not suited for quarks inside hadrons), poses a significant computational challenge. Thus, we are only able to reach a finite shell number in the three-body problem.

In exchange, our Hamiltonian matrix is relatively small and its diagonalization straightforward.

In the next sections the reader will find an exposé of why chiral symmetry has been expected to be manifest in the high hadron spectrum (Sec. II); a short discussion of why tracking the possibly decreasing Yrast parity splittings in the high spectrum is interesting to access the running quark mass function (Sec. III); a brief discussion of the model field-theory Hamiltonian and its variational treatment 
(Sec. IV); the detailed construction of the three-quark variational wave function basis (Sec. V); the report of our numerical results (Sec. VI); and a discussion thereof of the mechanisms that may be slowing the convergence to the expected parity doubling in the high baryon spectrum by breaking chiral symmetry, which deserves future investigation (Sec. VII). A small Appendix is dedicated to a somewhat technical computation of exchange matrix elements needed for the wave function overlaps that appear in antisymmetrizing and orthonormalizing the variational basis.

\section{INSENSITIVITY TO CHIRAL SYMMETRY BREAKING IN THE HIGH HADRON SPECTRUM}

Here, in Sec. II A we quickly revise the argument suggesting parity doubling in the high spectrum, and in the next section, Sec. II B, we show that one could conceivably use this possible doubling, if experimentally found, to make a statement about the running quark mass.

\section{A. Three-quark chiral (nondegenerate) quartet and its two (possibly degenerate) doublets}

Baryon valence wave functions ${ }^{1}$ with three quarks $\sum_{i j k} F_{i j k} B_{i}^{\dagger} B_{j}^{\dagger} B_{k}^{\dagger}|\Omega\rangle$ naturally group into reducible, nondegenerate quartets high in the spectrum, with two states having positive parity and two states having negative parity, and this quartet is split into two doublets. We frame the discussion within standard Hamiltonian techniques without invoking yet any particular model; in Sec. IV below we are more specific and introduce the actual Hamiltonian that is treated numerically.

Following the concise overview of Jaffe et al. [58], let us recall the possible representations of chiral symmetry in the hadron spectrum. In Wigner-Weyl mode, the commutator of the chiral charge $Q_{5}^{a}$ with a positive parity baryon field is a sum over negative parity baryons and vice versa,

$$
\begin{aligned}
& {\left[Q_{5}^{a}, \sigma_{i}^{+}\right]=\Theta_{i j}^{a} \sigma_{j}^{-}} \\
& {\left[Q_{5}^{a}, \sigma_{i}^{-}\right]=\Theta_{i j}^{a} \sigma_{j}^{+} .}
\end{aligned}
$$

Wigner's theorem then guarantees that if $\left[Q_{5}^{a}, H\right]=0$ (as is the case for the $\gamma^{0} \gamma^{0}$ Coulomb-type kernel in Sec. IV below and more generally in chromodynamics), and the vacuum state is unique, $Q_{5}^{a}|\Omega\rangle=0$, then $\sigma^{+}$and $\sigma^{-}$are degenerate.

However, if the symmetry is spontaneously broken (or hidden), a Goldstone boson appears, the pion, and chiral symmetry is realized nonlinearly. Because the pion has negative parity,

\footnotetext{
${ }^{1}$ We follow standard notation and use the greek indices $\lambda, \mu, \nu \ldots$ for spin, latin $k, p$ and $q$ for momenta, $f$ for flavor, $a$ for isospin, $c$ for color, and generically $i, j, k$ for excitations with the same quantum numbers (in baryons) or if we wish to be unspecific (in quarks).
}

$$
\left[Q_{5}^{a}, \sigma_{i}^{ \pm}\right]=v_{0}\left(\pi^{2}\right) \epsilon_{a b c} \pi^{c} \Theta_{i j}^{b} \sigma_{j}^{ \pm},
$$

where $v_{0}$ is a scalar function (e.g., $\left.v_{0}=1\right)$ of the pion field.

We now descend to the quark level, in the spirit of Nefediev et al. [23]. The chiral charge is the spatial integral of the zeroth component of the axial current,

$$
Q_{5}^{a}=\int d^{3} x \Psi^{\dagger}(x) \gamma_{5} \frac{\tau^{a}}{2} \Psi(x)
$$

We transform the fields to momentum space in a Bogoliubov-rotated basis

$$
\Psi(\mathbf{x})=\int \frac{\mathrm{d}^{3} k}{(2 \pi)^{3}} e^{i \mathbf{k} \cdot \mathbf{x}} \sum_{\lambda i}\left[U_{k \lambda} B_{k \lambda i}+V_{-k \lambda} D_{-k \lambda i}^{+}\right]
$$

in terms of the spinors,

$$
\begin{gathered}
U_{k \lambda}=\frac{1}{\sqrt{2}}\left[\begin{array}{c}
\sqrt{1+\sin \phi_{k}} \chi_{\lambda} \\
\sqrt{1-\sin \phi_{k}} \cdot \hat{k} \chi_{\lambda}
\end{array}\right], \\
V_{-k \lambda}=\frac{1}{\sqrt{2}}\left[\begin{array}{c}
-\sqrt{1-\sin \phi_{k}} \sigma \cdot \hat{k} i \sigma_{2} \chi_{\lambda} \\
\sqrt{1+\sin \phi_{k}} i \sigma_{2} \chi_{\lambda}
\end{array}\right],
\end{gathered}
$$

written as functions of the chiral angle with $\sin \phi(k)=$ $m(k) / \sqrt{m(k)^{2}+k^{2}}$ (the $k$ dependence is absent in this parametrization for free spinors: ours are more general and useful in the interacting theory).

There are four possible terms after normal ordering the charge in Eq. (2.3), $B^{\dagger} B, B^{\dagger} D^{\dagger}, D^{\dagger} D, D B$. The last two vanish when the chiral charge acts on an initial baryon state made of three quarks $|\sigma\rangle=\sum F B^{\dagger} B^{\dagger} B^{\dagger}|\Omega\rangle$ since the antiquark destruction operator $D$ acts directly on the vacuum and gives 0 . After spinor contractions,

$$
\begin{aligned}
Q_{a}^{5}|\sigma\rangle= & \int \frac{d^{3} k}{(2 \pi)^{3}} \sum_{\lambda \lambda^{\prime} f f^{\prime} c}\left(\frac{\tau^{a}}{2}\right)_{f f^{\prime}} \\
& \times\left(\cos \phi(k)(\sigma \cdot \hat{\mathbf{k}})_{\lambda \lambda^{\prime}} B_{k \lambda f c}^{\dagger} B_{k \lambda^{\prime} f^{\prime} c}\right. \\
& \left.+\sin \phi(k)\left(i \sigma_{2}\right)_{\lambda \lambda^{\prime}} B_{k \lambda f c}^{\dagger} D_{-k \lambda^{\prime} f^{\prime} c}^{\dagger}\right)|\sigma\rangle .
\end{aligned}
$$

The first term conserves the quark number and therefore remains within the same variational subspace. The second, however, creates a quark-antiquark pair on the baryon wave function. It is well known that the wave function associated with this pair, $\sin \phi(k) i \sigma_{2}$, precisely corresponds to a pion creator operator in Random Phase Approximation (and more generally it is an adequate pion interpolating field due to its quantum numbers). Therefore, this second term is responsible for the nonlinear realization of the symmetry: if chiral symmetry is spontaneously broken, $m(k) \neq 0$; thus $\sin \phi(k) \neq 0$ and under a chiral rotation a baryon is mapped to a baryon plus a pion $\sigma \rightarrow \pi \sigma$. 


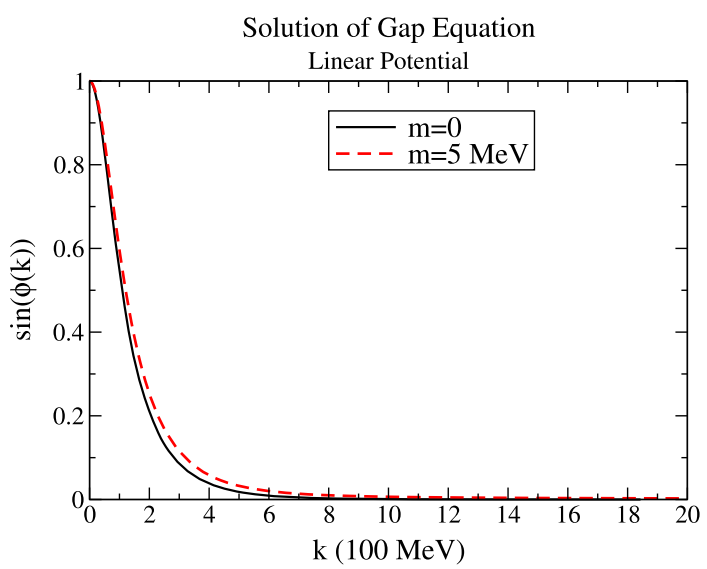

FIG. 3. Solution of the gap equation, $\sin \phi(k)$, for the linear potential with Coulomb-gauge-type $\gamma_{0} \gamma_{0}$ vector coupling. The current quark mass is fixed at $m_{u}=5 \mathrm{MeV}$ at a high scale.

Now, if the wave function $F\left(k_{i}, k_{j}, k_{l}\right)$ has its support for very large values of the momenta, then $m(k) \simeq 0$ there, since the running quark mass is large precisely for small values of the momentum (see Fig. 3 below). This means that there is a chance that chiral symmetry is realized in the Wigner-Weyl mode in the high spectrum. This would happen because the second term in Eq. (2.7) is now small, so chiral symmetry is realized linearly in the number of quarks by the first term of Eq. (2.7) that returns Eq. (2.1).

Once chiral symmetry becomes realized linearly in the Wigner-Weyl mode, degenerate baryon multiplets appear. The three-quark wave functions group in quartets that can be immediately found by successively applying the charge $Q_{5}^{a}$ to a given three-quark wave function.

Employing only one quark index $i$, for the case of one flavor, and showing the parity of the states, we can very transparently list the four linearly independent vectors for the quartet, ${ }^{2}$

$$
\begin{aligned}
\left|\sigma_{1}^{P}\right\rangle & =\sum F_{i j k}^{P} B_{i}^{\dagger} B_{j}^{\dagger} B_{k}^{\dagger}|\Omega\rangle, \\
\left|\sigma_{2}^{-P}\right\rangle= & \sum F_{i j k}^{P}\left(\sigma \cdot \hat{\mathbf{k}}_{i} \frac{\tau^{a}}{2} B^{\dagger}\right)_{i} B_{j}^{\dagger} B_{k}^{\dagger}|\Omega\rangle, \\
\left|\sigma_{3}^{P}\right\rangle= & \sum F_{i j k}^{P}\left(\sigma \cdot \hat{\mathbf{k}}_{i} \frac{\tau^{a}}{2} B^{\dagger}\right)_{i}\left(\sigma \cdot \hat{\mathbf{k}}_{j} \frac{\tau^{b}}{2} B^{\dagger}\right)_{j} B_{k}^{\dagger}|\Omega\rangle, \\
\left|\sigma_{3}^{-P}\right\rangle= & \sum F_{i j k}^{P}\left(\sigma \cdot \hat{\mathbf{k}}_{i} \frac{\tau^{a}}{2} B^{\dagger}\right)_{i}\left(\sigma \cdot \hat{\mathbf{k}}_{j} \frac{\tau^{b}}{2} B^{\dagger}\right)_{j} \\
& \times\left(\sigma \cdot \hat{\mathbf{k}}_{k} \frac{\tau^{c}}{2} B^{\dagger}\right)_{k}|\Omega\rangle ;
\end{aligned}
$$

the sequence arises by acting over the three quarks of $\left|\sigma_{1}^{P}\right\rangle$ in the first line. The baryon charge is the sum of three

\footnotetext{
${ }^{2} F$ is antisymmetric in its three indices because the fermion $B$ operators anticommute.
}

components, one for each quark $Q_{5}^{a}=q_{51}^{a}+q_{52}^{a}+q_{53}^{a}$, each of them reversing the spin projection over the quark momentum axis with the matrix $\sigma \cdot \hat{\mathbf{k}}_{i}$. Repeated application of a charge over the same quark does not change the parity since $\left(\sigma \cdot \hat{\mathbf{k}}_{i}\right)^{2}=\rrbracket$. Therefore, successive application generates new terms by rotating different quarks.

The total angular momentum $J$ of the four states coincides because the chiral charge is a pseudoscalar operator, so that $\left[J, Q_{5}^{a}\right]=0$. Each member of this chiral quartet is itself a multiplet under isospin transformations (e.g., a proton-neutron doublet).

Moreover, the isospin Pauli matrices $\tau^{a}$ produce tensor isospin operators that may mix the nucleon $I=1 / 2$ spectrum with the delta $I=3 / 2$ spectrum [8-10,12]. Thus we are interested in studying possible multiplets with the Yrast and Yrare (ground state and first excitation) with parity + and - and isospin $1 / 2$ and $3 / 2$.

\section{B. Series expansion in $m(k) / k$}

We now try to track the size of the chiral symmetry breaking effect of the dynamical quark mass in the high spectrum. The quark mass appears both in the quark spinors and in the QCD Hamiltonian. As we wish to track what happens for small quark mass at high momenta, we pursue a series expansion of the spinors and Hamiltonian in the parameter $m(k) / k$.

The spinors are expanded as

$$
\begin{gathered}
U_{k \lambda}=\frac{1}{\sqrt{2 E(k)}}\left[\begin{array}{c}
\sqrt{E(k)+m(k)} \chi_{\lambda} \\
\sqrt{E(k)-m(k)} \sigma \cdot \hat{k} \chi_{\lambda}
\end{array}\right] \\
\stackrel{\mathrm{k} \rightarrow \infty}{\frac{1}{\sqrt{2}}}\left[\begin{array}{c}
\chi_{\lambda} \\
\sigma \cdot \hat{k} \chi_{\lambda}
\end{array}\right]+\frac{1}{2 \sqrt{2}} \frac{m(k)}{k}\left[\begin{array}{c}
\chi_{\lambda} \\
-\sigma \cdot \hat{k} \chi_{\lambda}
\end{array}\right],
\end{gathered}
$$

with $E(k)=\sqrt{k^{2}+m(k)^{2}}$. Therein we have worked up to the leading chiral symmetry breaking term, which must be $O(m(k) / k)$.

When expanding the matrix elements of the QCD Hamiltonian [59] restricted to the Hilbert space of highly excited resonances where $\langle k\rangle$ is large, the zeroth order term in the $m(k) / k$ expansion is chirally invariant, and the firstorder term involves nonchiral, spin-dependent potentials in the quark-quark interaction,

$$
\begin{aligned}
& \left\langle n_{1}\left|H^{\mathrm{QCD}}\right| n_{2}\right\rangle \\
& \quad \simeq\left\langle n_{1}\left|H_{\chi}^{\mathrm{QCD}}\right| n_{2}\right\rangle+\left\langle n_{1}\left|\frac{m(k)}{k} H_{\chi}^{\mathrm{QCD}}\right| n_{2}\right\rangle \ldots
\end{aligned}
$$

Importantly, the lower spinor component of the first-order term has the opposite sign of the lower component of the chiral invariant term preceding it in the second line of Eq. (2.9).

With massless quarks, we have a vanishing commutator [23] $\left\langle i\left|\left[Q_{5}^{a}, H_{\mathrm{QCD}}\right]\right| j\right\rangle=0$ in the perturbative basis; but 
chiral noninvariant mass terms appear in the strong interactions due to dynamical mass generation, with chiral symmetry spontaneously broken by the ground state, $Q_{5}^{a}|0\rangle \neq 0$, a large quark mass in the propagator, pseudo-Goldstone bosons, and the loss of parity degeneracy in ground-state baryons. It appears that in the hadron basis $\left\langle n_{1}\left|\left[Q_{5}^{a}, H_{\mathrm{QCD}}\right]\right| n_{2}\right\rangle \neq 0$.

To discuss this situation, we write the chiral charge once more, now in terms of $m(k)$ instead of $\sin \phi(k)$,

$$
\begin{aligned}
Q_{5}^{a}= & \int \frac{d^{3} k}{(2 \pi)^{3}} \sum_{\lambda \lambda^{\prime} f f^{\prime} c}\left(\frac{\tau^{a}}{2}\right)_{f f^{\prime}} \frac{k}{\sqrt{k^{2}+m^{2}(k)}} \\
& \times\left[(\sigma \cdot \hat{\mathbf{k}})_{\lambda \lambda^{\prime}}\left(B_{k \lambda f c}^{\dagger} B_{k \lambda^{\prime} f^{\prime} c}+D_{-k \lambda^{\prime} f^{\prime} c}^{\dagger} D_{-k \lambda f c}\right)\right. \\
& \left.+\frac{m(k)}{k}\left(i \sigma_{2}\right)_{\lambda \lambda^{\prime}}\left(B_{k \lambda f c}^{\dagger} D_{-k \lambda^{\prime} f^{\prime} c}^{\dagger}+B_{k \lambda^{\prime} f^{\prime} c} D_{-k \lambda f c}\right)\right] .
\end{aligned}
$$

The first term between the square brackets once more consists of quark and antiquark number operators flipping spin and parity. The observation is that for $m(k) \ll k$, it dominates over the second term, which creates or annihilates a pion (realizing chiral symmetry nonlinearly). Thus, in the high-lying spectrum,

$$
\left\langle n_{1}\left|\left[Q_{5}^{a}, H_{\mathrm{QCD}}\right]\right| n_{2}\right\rangle \simeq 0 \text { for large } n_{1}, n_{2}
$$

and each parity doublet appears to become degenerate when $m(k)$ vanishes. Moreover, the mass splitting between partners is a direct measure of $m(k)$.

\section{THE RUNNING QUARK MASS AND THE $\Delta, N$ SPECTRA}

Equation (2.12) permits one to link the mass splitting $\left|M^{P=+}-M^{P=-}\right|$ in a parity doublet to the running quark mass. We concentrate on the lowest-lying $N$ and $\Delta$ parity doublets for increasing spin $j$ (the Yrast baryons) and reason as follows.

(1) Baryon masses fall on Regge trajectories,

$$
j=\alpha_{0}+\alpha M^{ \pm 2} \stackrel{j \rightarrow \infty}{\rightarrow} \alpha M^{ \pm 2},
$$

so that we can track the gross behavior of baryon masses with angular momentum.

(2) The relativistic virial theorem [60] in a few-body system at fixed particle number,

$$
\langle k\rangle \rightarrow c_{2} M^{ \pm} \rightarrow \frac{c_{2}}{\sqrt{\alpha}} \sqrt{j},
$$

shows how the average momentum grows in the high spectrum. (Here $c_{2}$ is an unspecified constant.)

(3) The chirally invariant term $\left(\left\langle n\left|H_{\chi}^{\mathrm{QCD}}\right| n\right\rangle\right)$ of Eq. (2.10) cancels out in the splitting $\Delta M$ so that for small $m(k) / k$,

$$
\left|M^{+}-M^{-}\right| \ll M^{ \pm}
$$

and

$$
\begin{aligned}
\left|M^{+}-M^{-}\right| & \rightarrow\left\langle\frac{m(k)}{k} H_{\chi}^{\mathrm{QCD} \prime}\right\rangle \\
& \rightarrow c_{3} \frac{m(k)}{k}\left\langle H_{\chi}^{\mathrm{QCD} \prime}\right\rangle .
\end{aligned}
$$

(4) In the first nonvanishing term in the splitting, $H_{\chi}^{\mathrm{QCD}}$, the spin-orbit $\mathbf{L}_{i} \cdot \mathbf{S}_{i}$ term transforms the angular momentum in the centrifugal barrier term from $\mathbf{L}_{i}^{2}$ to the chirally invariant $\mathbf{L}_{i}^{2}+2 \mathbf{L}_{i} \cdot \mathbf{S}_{i}=\mathbf{J}_{i}^{2}-\frac{3}{4}$. (Because of the sign difference in the helicity-dependent term $\sim-\boldsymbol{\sigma} \cdot \hat{\mathbf{k}}$ in the spinor, the spin-orbit term in $H_{\chi}^{\mathrm{QCD} \prime}$ adds to the mass difference $\Delta M$, instead of canceling out as for $H_{\chi}^{\mathrm{QCD}}$.) Thus, the centrifugal barrier scales like the mass of the state itself $M^{ \pm}$for high $j$, as per Eq. (3.1). The spin-orbit term on the other hand, $\mathbf{L}_{i} \cdot \mathbf{S}_{i} \sim J_{i}$, scales with one less power of $j$, and so the term producing the parity splitting actually decreases,

$$
\left\langle H_{\chi}^{\mathrm{QCD} \prime}\right\rangle \rightarrow c_{5} \frac{M^{ \pm}}{j} \rightarrow \frac{c_{5}}{\sqrt{\alpha}} \sqrt{\frac{1}{j}} .
$$

Combining these four arguments, we obtain

$$
\left|M^{+}-M^{-}\right| \rightarrow \frac{c_{3} c_{5}}{c_{2} \sqrt{\alpha}} m(\langle k\rangle) j^{-1} .
$$

An experimental extraction can fit the exponent $-i$ of $j$ in the splitting

$$
\left|M^{+}-M^{-}\right| \propto j^{-i} .
$$

Then an experimental extraction of the power-law behavior of the running quark mass in the region of chiral symmetry breaking is given by

$$
m(k) \propto k^{-2 i+2} .
$$

Returning to Fig. 1, we see that the experimentally known splittings of lowest-lying $N$ and $\Delta$ resonances are insufficient to derive the exponent $i$.

Knowledge of the masses of the parity doublets for spins $j>9 / 2$ would greatly enhance this (typically, only the natural parity mass is known above this, and all partners are missing from the tables).

The beauty of this application of the would-be doublets is that it directly maps experimental data to an underlying QCD property, here the exponent of the running quark 
mass. The numerical value of the mass itself, being gauge dependent, is not accessible. But the exponent of its powerlaw running at midmomentum, related to chiral symmetry breaking, could well be, as other symmetry-related quantities are (for example, the quark spin is accessible in deeply inelastic scattering). If the exponent is indeed gauge independent, then we should be able to access it in lattice gauge theory, and indeed one of us has already found some indications in heavy-light mesons [22].

It is interesting to note that a whole different way to access the running mass function from experiment is through the study of electromagnetic form factors $[61,62]$ where, due to Ward identities, the hadron wave functions are related to the running quark mass, so that certain integrated moments thereof can be probed.

\section{MODEL HAMILTONIAN}

Our so far generic arguments make it compelling to look for the parity doublets in the high baryon spectrum. Yet we wish to have a complete model computation at hand to thoroughly explore the physics. After an intense effort, we have now carried the program out and report it in this article.

We employ a well-known Hamiltonian density [18,24-31] that respects all global symmetries of QCD, and particularly chiral symmetry that is only broken spontaneously.

We adopt the truncated Coulomb-gauge formulation of QCD, also equivalent to the field-theory upgrade of the Cornell model,

$$
\begin{aligned}
\mathcal{H}(\mathbf{x})= & \Psi^{\dagger}(\mathbf{x})(-i \alpha \cdot \nabla+m \beta) \Psi \\
& -\frac{1}{2} \int \mathrm{d}^{3} y \Psi^{\dagger}(\mathbf{x}) T^{a} \Psi(\mathbf{x}) V(\mathbf{x}-\mathbf{y}) \Psi^{\dagger}(\mathbf{y}) T^{a} \Psi(\mathbf{y}) ;
\end{aligned}
$$

here $T^{a}$ is Gell-Mann's color matrix, and the field operator is expanded as in Eq. (2.4) above to obtain the momentumspace representation. Moreover, we consider the case of a linearly confining Coulomb term, necessary for linear Regge trajectories.

This model has been extensively discussed in previous literature [63], so we recapitulate only the minimal set of features that we need.

\section{A. Gap equation and numerical solution}

Minimizing the vacuum expectation value of the Hamiltonian in the BCS approximation yields a gap equation for $\sin \phi(k)$ (the sine and the cosine are shortened to $s_{k}$ and $c_{k}$ as needed). This has been solved for the linear potential in the past $[32,52,53]$ and is known to have a tower of symmetry breaking solutions. The ground-state solution is a monotonous function of $k$ and has been obtained along standard methods $[64,65]$ by solving the nonlinear integral equation on a computer. It is shown in
Fig. 3. The chiral angle enters the later computation through the three-quark matrix elements of the Hamiltonian in Eq. (4.5).

The BCS gap function for the linear potential, resulting solely from the Hamiltonian density in Eq. (4.1), yields a small constituent quark mass of order $100 \mathrm{MeV}$, already parametrized in excellent approximation by the ansatz [65],

$$
\mathcal{A}_{3}(p)=m_{0}+\frac{1}{c_{0}+c_{2} p^{2}+c_{4} p^{4}},
$$

with the vacuum minimization parameters, $c_{0}=6.01623$, $c_{2}=23.2517, c_{4}=12.0965$ in dimensionless units of the meson string tension $\frac{4}{3} \sigma=1$, for a bare mass of $m_{0}=0.01 \sqrt{\frac{4}{3} \sigma} \simeq 4 \mathrm{MeV}$. A more complete model for the truncated Coulomb-gauge potential, with more parameters, is necessary to get a larger constituent quark mass. As a sanity check we have also employed a constant quark mass $m(k) \rightarrow m(0)$. In Ref. [1] the mass gap function generated in Coulomb-gauge SU(2) lattices [66], as well as in Landau gauge SU(3) lattices [67], was also used. There are of course differences of detail from using different masses, but not of principle.

High in the spectrum, various relativistic splittings are suppressed and the onset of chiral restoration could be masked by other effects. Therefore, we need a benchmark calculation to know the quantitative amount of insensitivity to chiral symmetry breaking.

The litmus test for whether the parity partners are becoming degenerate due to chiral symmetry is passed if the energy difference between doublers is significantly smaller for the chiral theory.

Moreover, we use our same computer code but fix $m(k)=M$ to a constant (like in the constituent model). This means $\sin \phi(k)=1$ in that benchmark calculation, which is reported below in Fig. 11 next to other systematic analysis. This allows one to estimate whether the running mass can be extracted from the spectrum, as discussed in Sec. II B.

\section{B. Exposing parity doubling within the model}

The complete three-quark baryon wave function given below in Eqs. (5.1) and (5.3) can be shortened to

$|\sigma\rangle=\frac{\epsilon^{i j k}}{\sqrt{6}} F_{\sigma}^{\lambda_{1} \lambda_{2} \lambda_{3}}\left(\mathbf{k}_{1}, \mathbf{k}_{2}, \mathbf{k}_{3}\right) B_{\mathbf{k}_{1} \lambda_{1} i}^{\dagger} B_{\mathbf{k}_{2} \lambda_{2} j}^{\dagger} B_{\mathbf{k}_{3} \lambda_{3} k}^{\dagger}|\Omega\rangle$.

The momentum part of the wave function is

$$
\begin{aligned}
& F_{\sigma}^{\lambda_{1} \lambda_{2} \lambda_{3}}\left(\mathbf{k}_{1}, \mathbf{k}_{2}, \mathbf{k}_{3}\right) \\
& =e^{i \mathbf{K} \cdot \mathbf{R}} F_{\sigma}^{\lambda_{1} \lambda_{2} \lambda_{3}}\left(\rho\left(\mathbf{k}_{1}, \mathbf{k}_{2}, \mathbf{k}_{3}\right), \lambda\left(\mathbf{k}_{1}, \mathbf{k}_{2}, \mathbf{k}_{3}\right)\right),
\end{aligned}
$$

with $\mathbf{K}$ and $\mathbf{R}$ being the total momentum and center of mass coordinate, and $\rho, \lambda$ the Jacobi coordinates (see Sec. VA below). 
The resulting matrix elements of the Hamiltonian in Eq. (4.1) are then [68]

$$
\begin{aligned}
\left\langle\sigma|H| \sigma^{\prime}\right\rangle= & \delta^{3}\left(\mathbf{R}-\mathbf{R}^{\prime}\right) 3 \int \frac{\mathrm{d}^{3} k_{1}}{(2 \pi)^{3}} \frac{\mathrm{d}^{3} k_{2}}{(2 \pi)^{3}}\left[F_{\sigma}^{\lambda_{1} \lambda_{2} \lambda_{3}}\left(\mathbf{k}_{1}, \mathbf{k}_{2}\right)\right]^{*}\left\{F_{\sigma^{\prime}}^{\lambda_{1} \lambda_{2} \lambda_{3}}\left(\mathbf{k}_{1}, \mathbf{k}_{2}\right)\left(m s_{k_{1}}+\left|\mathbf{k}_{1}\right| c_{k_{1}}\right)-\frac{2}{3} \int \frac{\mathrm{d}^{3} q}{(2 \pi)^{3}} V(|\mathbf{q}|)\right. \\
& \left.\times\left[F_{\sigma^{\prime}}^{\lambda_{1} \lambda_{2} \lambda_{3}}\left(\mathbf{k}_{1}, \mathbf{k}_{2}\right)\left(s_{k_{1}} s_{k_{1}+q}+c_{k_{1}} c_{k_{1}+q} \hat{\mathbf{k}_{1}} \cdot \widehat{\mathbf{k}_{1}+\mathbf{q}}\right)-F_{\sigma^{\prime}}^{\mu_{1} \mu_{2} \lambda_{3}}\left(\mathbf{k}_{1}+\mathbf{q}, \mathbf{k}_{2}-\mathbf{q}\right)\left(U_{\mathbf{k}_{1} \lambda_{1}}^{+} U_{\mathbf{k}_{1}+\mathbf{q} \mu_{1}}\right)\left(U_{\mathbf{k}_{2} \lambda_{2}}^{+} U_{\mathbf{k}_{2}-\mathbf{q} \mu_{2}}\right)\right]\right\} .
\end{aligned}
$$

Here, the arguments of the wave functions $F$ are the momenta of the first and second quark, and should be transformed into Jacobi momenta in the rest frame $(\mathbf{K}=\mathbf{0})$. Furthermore, sums over all spin quantum numbers are assumed. The integrals to compute are nine dimensional, but those over $\mathbf{q}$ can be performed very efficiently. Additionally, the use of this variable diminishes numerical instabilities associated with the interaction, $V(|\mathbf{q}|)=\frac{-8 \pi \sigma}{q^{4}}$, the linear string potential in momentum space, that has a strong infrared divergence. That denominator is indeed partly offset by the $q^{2}$ Jacobian factor if this $q$ variable is chosen for integration. Moreover, both one-body and two-body potential terms in the second line of Eq. (4.5) can be expanded in powers of $\mathbf{q}$, and the zeroth order terms cancel (a chiral Ward identity). The next order term is proportional to $\mathbf{q}$ and is eliminated by the angular integration, leaving the $q^{2}$ term as the lowest order term on the expansion of the difference. This and the Jacobian factor cancel the infrared $1 / q^{4}$ divergence. Therefore, the integrand is regular and no regulator is needed. Furthermore, we have verified that there is no instability in the numerical results.

Let us now see how the mechanism for insensitivity to chiral symmetry breaking discussed in generic terms heretofore is at work for the simplified Hamiltonian in Eq. (4.1) above. That the kinetic energy is invariant under the spin rotation caused by the $\sigma \cdot \hat{\mathbf{k}}$ in Eq. (2.11) is obvious. Indeed, if the first state of the quartet in Eq. (2.8) has as a wave function

$$
|1\rangle=F^{s_{1} s_{2} s_{3}}\left(\mathbf{k}_{1}, \mathbf{k}_{2}\right),
$$

its kinetic and self-energies in Eq. (4.5) are proportional to the simple overlap

$$
F^{s_{1} s_{2} s_{3} \dagger}\left(\mathbf{k}_{1}, \mathbf{k}_{2}\right) F^{s_{1} s_{2} s_{3}}\left(\mathbf{k}_{1}, \mathbf{k}_{2}\right),
$$

and if we take as the second element of the quartet that with only one spin rotation, we have

$$
|2\rangle=\left(\sigma \cdot \hat{\mathbf{k}}_{1}\right)_{s_{1} s_{1}^{\prime}} F^{s_{1}^{\prime} s_{2} s_{3}}\left(\mathbf{k}_{1}, \mathbf{k}_{2}\right),
$$

and its kinetic energy and self-energy are then proportional to ( $\sigma$ being Hermitian)

$F^{s_{1}^{\prime} s_{2} s_{3} \dagger}\left(\mathbf{k}_{1}, \mathbf{k}_{2}\right)\left(\sigma \cdot \hat{\mathbf{k}}_{1}\right)_{s_{1}^{\prime} s_{1}}\left(\sigma \cdot \hat{\mathbf{k}}_{1}\right)_{s_{1} s_{1}^{\prime \prime}} F_{1}^{s_{1}^{\prime} s_{2} s_{3}}\left(\mathbf{k}_{1}, \mathbf{k}_{2}\right) ;$ and since $\sigma \cdot \hat{\mathbf{k}}_{1} \sigma \cdot \hat{\mathbf{k}}_{1}=\llbracket$ the terms in (4.7) and (4.9) are identical.

Moving on to the potential energy in Eq. (4.5), degeneracy requires the following equality to hold (irrelevant parts of $\langle V\rangle$ are omitted):

$$
\begin{aligned}
F^{\lambda_{1} \lambda_{2} \lambda_{3} \dagger}\left(\mathbf{k}_{1}, \mathbf{k}_{2}\right)\left(U_{\mathbf{k}_{1} \lambda_{1}}^{+} U_{\mathbf{k}_{1}+\mathbf{q} \mu_{1}}\right) F^{\mu_{1} \mu_{2} \lambda_{3}}\left(\mathbf{k}_{1}+\mathbf{q}, \mathbf{k}_{2}-\mathbf{q}\right) \\
=F^{\lambda_{1}^{\prime} \lambda_{2} \lambda_{3} \dagger}\left(\mathbf{k}_{1}, \mathbf{k}_{2}\right)\left(\sigma \cdot \hat{\mathbf{k}}_{1}\right)_{\lambda_{1}^{\prime} \lambda_{1}}\left(U_{\mathbf{k}_{1} \lambda_{1}}^{+} U_{\mathbf{k}_{1}+\mathbf{q} \mu_{1}}\right) \\
\quad \times\left(\sigma \cdot\left(\widehat{\mathbf{k}_{1}+\mathbf{q}}\right)\right)_{\mu_{1} \mu_{1}^{\prime}} F^{\mu_{1}^{\prime} \mu_{2} \lambda_{3}}\left(\mathbf{k}_{1}+\mathbf{q}, \mathbf{k}_{2}-\mathbf{q}\right) .
\end{aligned}
$$

Substituting the spinors in Eq. (4.5) this reduces to whether the following equality is or is not satisfied:

$$
\begin{aligned}
\sqrt{1+s_{k_{1}}} & \sqrt{1+s_{k_{1}+q}} \delta_{\lambda_{1} \mu_{1}}+\sqrt{1-s_{k_{1}}} \sqrt{1-s_{k_{1}+q}} \\
& \times\left(\sigma \cdot \hat{\mathbf{k}}_{1} \sigma \cdot\left(\widehat{\mathbf{k}_{1}+\mathbf{q}}\right)\right)_{\lambda_{1} \mu_{1}} \\
= & \left(\sigma \cdot \hat{\mathbf{k}}_{1}\right)_{s_{1}^{\prime} s_{1}}\left(\sqrt{1+s_{k_{1}}} \sqrt{1+s_{k_{1}+q}} \delta_{s_{1} \lambda_{1}}\right. \\
& +\sqrt{1-s_{k_{1}}} \sqrt{1-s_{k_{1}+q}}\left(\sigma \cdot \hat{\mathbf{k}}_{1} \sigma \cdot\left(\widehat{\mathbf{k}_{1}+\mathbf{q}}\right)\right)_{s_{1} \lambda_{1}} \\
& \times\left(\sigma \cdot\left(\widehat{\mathbf{k}_{1}+\mathbf{q}}\right)\right)_{\lambda_{1} \lambda_{1}^{\prime}} .
\end{aligned}
$$

The equality does not generally hold and the two states $|1\rangle,|2\rangle$ are not degenerate if chiral symmetry is broken. But high in the spectrum where the $F$ wave functions have support, $\sin \phi(k)$ is effectively 0 . Substituting $s_{k}=0$ in Eq. (4.11) and using $\sigma \cdot \hat{\mathbf{k}} \sigma \cdot \hat{\mathbf{k}}=\rrbracket$, it is satisfied. We tentatively conclude from this analysis that the Hamiltonian in Eq. (4.1) makes the two states degenerate when $\sin \phi(k) \rightarrow 0$ (but see later in Sec. VII).

The degeneracy would clearly be absent if we employed a chiral symmetry violating interaction with vertex $\bar{U} U=$ $U^{\dagger} \gamma_{0} U$ instead of the vector-coupled quarks in Eq. (4.1). In the Pauli-Dirac representation that we employ,

$$
\gamma_{0}=\left(\begin{array}{cc}
\square & 0 \\
0 & -\rrbracket
\end{array}\right)
$$

and hence 
$\left(\sigma \cdot \hat{\mathbf{k}}_{1}\right)_{s_{1} s_{1}^{\prime}}\left(U^{\dagger} \gamma_{0} U\right)_{s_{1}^{\prime} \lambda_{1}}\left(\sigma \cdot\left(\widehat{\mathbf{k}_{1}+\mathbf{q}}\right)\right)_{\lambda_{1} \lambda_{1}^{\prime}} \rightarrow-\left(U^{\dagger} \gamma_{0} U\right)_{s_{1}^{\prime} \lambda_{1}}$

when $\sin \phi(k) \rightarrow 0$, and there is no degeneracy. This demonstrates explicitly the cancellation in the chiral symmetric case, and the lack of cancellation in the chiral nonsymmetric case.

Beyond our simple model Hamiltonian, examination of the exact QCD one formulated in Coulomb gauge [59,69] immediately reveals that the differences lay in the gauge part but not in the quark-spin structure, and though the mass function $\sin \phi(k)$ may be chosen to involve more complicated correlations than the BCS ansatz, chiral symmetry appears so far to be manifested in the Wigner mode high in the spectrum.

To conclude this subsection, let us comment on the additional known consequence of possible insensitivity to chiral symmetry breaking in the high spectrum (a dynamical effect due to hadrons decoupling from the condensed fermions). This is that all resonances decouple from their $N \pi \ldots \pi$ decay channels; they have to decay through intermediate excited states (or if this is not possible, be very narrow). That is, $g_{N^{* *} N^{*} \pi} \rightarrow 0$ for all possible couplings involving pions. This is easy to see in the BCS approximation [23]: the coupling is proportional to a wave function overlap, formally

$$
g_{N^{* *} N^{*} \pi} \propto \int F^{N^{* *}} \mathcal{B}_{N^{*}} F^{N^{*}} \mathcal{B}_{\pi} \sin \phi(k),
$$

where the pion has been taken as an exact Goldstone boson with wave function $F^{\pi} \propto \sin \phi(k)$ and $\mathcal{B}$ are boost operators (as the outgoing wave functions correspond to moving particles). Both the boost operators and the very excited resonance wave function $F^{N^{* *}}$ in Eq. (4.14) select large values of the quark momentum $k$. This entails that $\sin \phi(k) \rightarrow 0$, and thus $g_{N^{* *} N^{*} \pi} \rightarrow 0$ too.

\section{VARIATIONAL WAVE FUNCTION BASIS}

The three-fermion problem is a classic of quantum mechanics (e.g., the triton nucleus), and extensive work has been carried out in the quark model for baryons $[46-51,70]$. We build on well-established results and put together a practical package of wave functions that can also be used in future applications. The construction of this basis and its numeric implementation is by far the most time consuming part of this project and we report it with some detail for reproducibility.

We can divide the construction of the wave function basis in several steps that are conceptually well separated and given in the following subsections. In essence, we start with the three-dimensional harmonic oscillator $(\mathrm{HO})$ for the intrinsic coordinates in momentum space, whose wave functions we denote by $\phi$. The spins need to be combined to yield states labeled by the conserved $J, m_{J}$. Then we antisymmetrize with respect to the three sets of quark quantum numbers, to yield a new set of three-body wave functions $\Phi$ that are orthonormal and antisymmetric. This is done with the help of the Moshinsky-Brody-Ribeiro-van Beveren (MBRB) coefficients [71-75].

\section{A. Momentum-space wave function and Jacobi coordinates}

As the first step, we employ a standard variational approximation to the vacuum, the BCS state $|\Omega\rangle$. A baryon, in lowest order in the Fock-space expansion, is a threequark excitation thereof,

$$
\begin{aligned}
|\mathcal{B}\rangle= & \sum_{c, s, f} \prod \int \frac{d^{3} p_{i}}{(2 \pi)^{3}} \frac{\epsilon^{c_{1} c_{2} c_{3}}}{\sqrt{6}} F_{\mathcal{B}}^{s, f}\left(\mathbf{p}_{1}, \mathbf{p}_{2}, \mathbf{p}_{3}\right) \\
& \times B_{c_{1}, s_{1}, f_{1}, \mathbf{p}_{1}}^{\dagger} B_{c_{2}, s_{2}, f_{2}, \mathbf{p}_{2}}^{\dagger} B_{c_{3}, s_{3}, f_{3}, \mathbf{p}_{3}}^{\dagger}|\Omega\rangle .
\end{aligned}
$$

We could use one-particle Cartesian momenta $\mathbf{p}_{1}$ and $\mathbf{p}_{2}$, while enforcing $\mathbf{p}_{3}=-\mathbf{p}_{1}-\mathbf{p}_{2}$. However, the possibility to use the algebraic MBRB coefficients that reduce the basis wave function overlap without need for any numerical integration prompted us to use the Jacobi coordinates. These, however, are not the standard nonrelativistic Jacobi coordinates that depend on the particle mass, since the relativistic kinetic energy is not quadratic and does not separate anyway. Because of symmetry considerations we choose instead

$$
\left(\begin{array}{l}
\mathbf{p}_{\rho} \\
\mathbf{p}_{\lambda} \\
\mathbf{p}_{R}
\end{array}\right)=\left(\begin{array}{ccc}
\frac{1}{\sqrt{2}} & -\frac{1}{\sqrt{2}} & 0 \\
\frac{1}{\sqrt{6}} & \frac{1}{\sqrt{6}} & -\frac{2}{\sqrt{6}} \\
\frac{1}{\sqrt{3}} & \frac{1}{\sqrt{3}} & \frac{1}{\sqrt{3}}
\end{array}\right)\left(\begin{array}{l}
\mathbf{p}_{1} \\
\mathbf{p}_{2} \\
\mathbf{p}_{3}
\end{array}\right)
$$

The color wave function $\frac{\epsilon^{c_{1} c_{2} c_{3}}}{\sqrt{6}}$ is normalized to 1 taking into account all possible contractions of the fields in the $\langle\mathcal{B} \mid \mathcal{B}\rangle$ overlap. Since we construct $F$ to be totally symmetric under exchange of any two quarks, we do not sum over permutations of the $B$ 's in the orthonormalization of the basis (to avoid double-counting).

In terms of the Jacobi coordinates, and in the center of mass $\mathbf{P}_{R}=0$ the wave function becomes

$$
F_{\mathcal{B}}^{s, f}\left(\mathbf{p}_{1}, \mathbf{p}_{2}, \mathbf{p}_{3}\right)=F_{\mathcal{B}}^{s, f}\left(\mathbf{p}_{\rho}, \mathbf{p}_{\lambda}\right) .
$$

\section{B. Single-particle harmonic oscillator wave functions}

For atomic physics systems, or weakly bound systems such as the deuteron, an adequate basis of functions is the Laguerre polynomial basis, decaying at large distances $e^{-c r}$. For particles in a box the Bessel functions are maybe optimal. In the present case of a linear confining potential, a practical basis is the eigenbasis of the HO. This is because 
many analytical results exist to simplify intermediate computations, and since the wave functions are confining, variational convergence for the linear potential should be straightforward. The more natural Airy basis (that diagonalizes the nonrelativistic Schrödinger equation with a linear potential) loses its advantage for relativistic kinetic energies, as necessary with a running mass function, and is more cumbersome to use.

Working in momentum space, we Fourier transform the HO functions, which depend on a parameter $\alpha$ with dimensions of length that scale with the size of the ground state. If we used only one or two basis elements, it would have to be interpreted as a variational parameter to be varied until a minimum was found. However, since we use an extended basis and diagonalize the Hamiltonian in it, $\alpha$ can be left fixed at any value (of course, an unreasonable value thereof leads to very poor convergence in the number of wave functions, so it is logical to take it of the order of $\left.\alpha \simeq 1 / \Lambda_{\mathrm{QCD}}\right)$. For each of the two $\mathbf{p}_{\rho}, \mathbf{p}_{\lambda}$, we have

$$
\begin{aligned}
\varphi_{n l m}^{\alpha}(\mathbf{r}) & =i^{2 n+l} \varphi_{n l}^{\alpha}(r) Y_{l m}(\hat{r}) \\
& =i^{2 n+l} \alpha^{-3 / 2} \varphi_{n l}\left(\alpha^{-1} r\right) Y_{l m}(\hat{r}) \\
\stackrel{F . T .}{\rightarrow} \varphi_{n l m}^{\alpha}(\mathbf{p}) & =(2 \pi \alpha)^{3 / 2} \varphi_{n l}(\alpha p) Y_{l m}(\hat{p}) .
\end{aligned}
$$

There being two independent three-dimensional variables $\mathbf{p}_{\rho}, \mathbf{p}_{\lambda}$, we take the product of two such HO functions, so that the unsymmetrized basis diagonalizes two independent harmonic oscillators. We take their two constants $\alpha_{\rho}=\alpha_{\lambda}=\alpha$ since this is useful to simplify the symmetrization of the wave functions on the variational basis. As just argued, the choice of $\alpha$ is only relevant for convergence speed. Doing so, the wave function space splits in shells of the sum of harmonic oscillators, each shell being characterized by a shell-quantum number

$$
N=\left(2 n_{\rho}+l_{\rho}+2 n_{\lambda}+l_{\lambda}\right) .
$$

It is useful to note that all states in the same shell have the same parity $P=(-1)^{N}=(-1)^{l_{\rho}+l_{\lambda}}$. Therefore, to study a baryon sector of given parity, only half the shells need to be kept (the three-quark intrinsic parities being positive).

For even parity, a shell with quantum number $N$ has $\frac{(N+2)(N+3)(N+4)}{24}$ independent degenerate (for the HO) spin multiplets of fixed $n_{\rho}, n_{\lambda}, l_{\rho}, l_{\lambda}$. Within each multiplet there are eight states corresponding to the two possible values of the spins $s_{1}, s_{2}, s_{3}$.

For odd parity, the corresponding number of multiplets is rather $\frac{(N+1)(N+3)(N+5)}{24}$. Thus, if we variationally limit the basis up to $N_{\max }$ shells, the number of multiplets (hence storage space in the computer, for example) grows asymptotically as $N_{\max }^{4}$.
TABLE I. The in-shell index $l$ within a certain oscillator shell $N$

\begin{tabular}{|c|c|c|c|c|c|}
\hline$N$ & $l$ & $n_{\rho}$ & $n_{\lambda}$ & $l_{\rho}$ & $l_{\lambda}$ \\
\hline 0 & 0 & 0 & 0 & 0 & 0 \\
\hline \multirow[t]{2}{*}{1} & 0 & 0 & 0 & 0 & 1 \\
\hline & 1 & 0 & 0 & 1 & 0 \\
\hline \multirow[t]{5}{*}{2} & 0 & 0 & 0 & 0 & 2 \\
\hline & 1 & 0 & 0 & 1 & 1 \\
\hline & 2 & 0 & 0 & 2 & 0 \\
\hline & 3 & 0 & 1 & 0 & 0 \\
\hline & 4 & 1 & 0 & 0 & 0 \\
\hline \multirow[t]{8}{*}{3} & 0 & 0 & 0 & 0 & 3 \\
\hline & 1 & 0 & 0 & 1 & 2 \\
\hline & 2 & 0 & 0 & 2 & 1 \\
\hline & 3 & 0 & 0 & 3 & 0 \\
\hline & 4 & 0 & 1 & 0 & 1 \\
\hline & 5 & 0 & 1 & 1 & 0 \\
\hline & 6 & 1 & 0 & 0 & 1 \\
\hline & 7 & 1 & 0 & 1 & 0 \\
\hline \multirow[t]{14}{*}{4} & 0 & 0 & 0 & 0 & 4 \\
\hline & 1 & 0 & 0 & 1 & 3 \\
\hline & 2 & 0 & 0 & 2 & 2 \\
\hline & 3 & 0 & 0 & 3 & 1 \\
\hline & 4 & 0 & 0 & 4 & 0 \\
\hline & 5 & 0 & 1 & 0 & 2 \\
\hline & 6 & 0 & 1 & 1 & 1 \\
\hline & 7 & 0 & 1 & 2 & 0 \\
\hline & 8 & 0 & 2 & 0 & 0 \\
\hline & 9 & 1 & 0 & 0 & 2 \\
\hline & 10 & 1 & 0 & 1 & 1 \\
\hline & 11 & 1 & 0 & 2 & 0 \\
\hline & 12 & 1 & 1 & 0 & 0 \\
\hline & 13 & 2 & 0 & 0 & 0 \\
\hline$\cdots$ & $\cdots$ & $\cdots$ & $\ldots$ & $\ldots$ & $\cdots$ \\
\hline
\end{tabular}
corresponds to a set of orbital quantum numbers $\left(n_{\rho}, n_{\lambda}, l_{\rho}, l_{\lambda}\right)$ as illustrated. There are degeneracies, e.g., $\left(2 l_{\rho}+1\right)\left(2 l_{\lambda}+1\right)$, that lift in subsequent steps.

Within a certain $\mathrm{HO}$ shell $N$, one can assign an in-shell quantum number or index (e.g., $l$ or $k$ ) to the different combinations of $\left(n_{\rho}, n_{\lambda}, l_{\rho}, l_{\lambda}\right)$ that make up $N$ according to Eq. (5.5). The one-to-one correspondence between the inshell index $l$ and the set of orbital quantum numbers is illustrated in Table I. Instead of writing the whole set of orbital quantum numbers, it is easier to refer to an $(N, l)$-pair.

\section{Spin coupling and spin index convention}

The wave functions of the variational basis include three $1 / 2$-spins, and the two orbital angular momenta, to yield the total angular momentum, recoupling with the help of Clebsch-Gordan (CG) coefficients, for which we use bra-ket notation $\left\langle l_{1} m_{1} l_{2} m_{2} \mid l m_{l}\right\rangle$. (The isospin coupling is barely discussed but to construct $I=1 / 2,3 / 2$ the procedure is the same, though simpler because of the orbital angular momentum complication here.) 
TABLE II. Labeling of the three uncoupled quark spins $\left(s_{1}, s_{2}, s_{3}\right)$ with a spin index $S_{i}$ for indexation in the computer.

\begin{tabular}{llll}
\hline \hline$S_{i}$ & $s_{1}$ & $s_{2}$ & $s_{3}$ \\
\hline 0 & $\uparrow$ & $\uparrow$ & $\uparrow$ \\
1 & $\uparrow$ & $\uparrow$ & $\downarrow$ \\
2 & $\uparrow$ & $\downarrow$ & $\uparrow$ \\
3 & $\uparrow$ & $\downarrow$ & $\downarrow$ \\
4 & $\downarrow$ & $\uparrow$ & $\uparrow$ \\
5 & $\downarrow$ & $\uparrow$ & $\downarrow$ \\
6 & $\downarrow$ & $\downarrow$ & $\uparrow$ \\
7 & $\downarrow$ & $\downarrow$ & $\downarrow$ \\
\hline \hline
\end{tabular}

The basis functions are made for a certain total spin $J$ and spin projection $M_{J}$. The basis is cut at a certain maximum value for the harmonic oscillator shell-quantum number $N_{\max }$. Ideally one would take the limit $N_{\max } \rightarrow \infty$ to make the variational treatment arbitrarily accurate, but this is not possible with finite computer power. In practice we have been able to reach $N_{\max }=12$ or less.

The starting set of basis functions with spin is simply chosen to be factorized,

$$
\begin{aligned}
& \mathcal{B}_{N, l, m_{\rho}, m_{\lambda}, R_{i}}^{\alpha}\left(\mathbf{p}_{\rho}, \mathbf{p}_{\lambda}, S_{i}\right) \\
& =\varphi_{n_{\rho}^{N, l}, l_{\rho}^{N, l}, m_{\rho}}^{\alpha}\left(\mathbf{p}_{\rho}\right) \varphi_{n_{\lambda}^{N, l}, l_{\lambda}^{N, l}, m_{\lambda}}^{\alpha}\left(\mathbf{p}_{\lambda}\right) \chi_{R_{i}}\left(S_{i}\right) .
\end{aligned}
$$

Here, the $\varphi$-functions are the harmonic oscillator wave functions from Eq. (5.4). The spin-dependent part of the basis function, $\chi_{R_{i}}\left(S_{i}\right)$, is basically a Kronecker-delta $\delta_{R_{i} S_{i}}$. The spin indices $R_{i}$ and $S_{i}$ are a shorthand notation for the three uncoupled quark spins according to Table II.

The set of basis functions (5.6) is to be recoupled to total $J$ and projection $M_{J}$. This is accomplished by constructing an array $\phi$ of coefficients that consist of a sum of products of Clebsch-Gordan coefficients that couple the quark spins and orbital quantum numbers to a fixed $\left(J, M_{J}\right)$. This changes the set of uncoupled quantum numbers,

$$
n_{\rho}^{N, l}, l_{\rho}^{N, l}, m_{\rho}, n_{\lambda}^{N, l}, l_{\lambda}^{N, l}, m_{\lambda}, S_{1}, s_{1}, S_{2}, s_{2}, S_{3}, s_{3},
$$

by the set of coupled quantum numbers,

$$
n_{\rho}^{\bar{N}, \bar{l}}, l_{\rho}^{\bar{N}, \bar{l}}, n_{\lambda}^{\bar{N}, \bar{l}}, l_{\lambda}^{\bar{N}, \bar{l}}, L^{\bar{N}}, S_{1}, S_{2}, S_{3}, S, S_{12}, J, M_{J}
$$

The barred ordering scheme for the coupled orbital quantum numbers is illustrated in Table III, and we use a bar to distinguish the indices specific to the coupled basis. The barred ordering scheme for the coupled spin quantum numbers is given in Table IV.
TABLE III. The barred shell number $\bar{N}$ with its barred in-shell index $\bar{l}$ corresponds to a set of orbital quantum numbers $\left(L, n_{\rho}, n_{\lambda}, l_{\rho}, l_{\lambda}\right)$ as illustrated. The unbarred shell number and in-shell index corresponding to the same set of orbital quantum numbers are also given. We remark that whenever an unbarred set

\begin{tabular}{|c|c|c|c|c|c|c|c|c|}
\hline $\bar{N}$ & $L$ & $\bar{l}$ & $n_{\rho}$ & $n_{\lambda}$ & $l_{\rho}$ & $l_{\lambda}$ & $N$ & $l$ \\
\hline 0 & 0 & 0 & 0 & 0 & 0 & 0 & 0 & 0 \\
\hline \multirow[t]{2}{*}{1} & 1 & 0 & 0 & 0 & 0 & 1 & 1 & 0 \\
\hline & & 1 & 0 & 0 & 1 & 0 & & 1 \\
\hline \multirow[t]{3}{*}{2} & 0 & 0 & 0 & 0 & 1 & 1 & 2 & 1 \\
\hline & & 1 & 0 & 1 & 0 & 0 & & 3 \\
\hline & & 2 & 1 & 0 & 0 & 0 & & 4 \\
\hline 3 & 1 & 0 & 0 & 0 & 1 & 1 & 2 & 1 \\
\hline \multirow[t]{3}{*}{4} & 2 & 0 & 0 & 0 & 0 & 2 & 2 & 0 \\
\hline & 2 & 1 & 0 & 0 & 1 & 1 & & 1 \\
\hline & 2 & 2 & 0 & 0 & 2 & 0 & & 2 \\
\hline \multirow[t]{6}{*}{5} & 1 & 0 & 0 & 0 & 1 & 2 & 3 & 1 \\
\hline & & 1 & 0 & 0 & 2 & 1 & & 2 \\
\hline & & 2 & 0 & 1 & 0 & 1 & & 4 \\
\hline & & 3 & 0 & 1 & 1 & 0 & & 5 \\
\hline & & 4 & 1 & 0 & 0 & 1 & & 6 \\
\hline & & 5 & 1 & 0 & 1 & 0 & & 7 \\
\hline \multirow[t]{2}{*}{6} & 2 & 0 & 0 & 0 & 1 & 2 & 3 & 1 \\
\hline & & 1 & 0 & 0 & 2 & 1 & & 2 \\
\hline \multirow[t]{4}{*}{7} & 3 & 0 & 0 & 0 & 0 & 3 & 3 & 0 \\
\hline & & 1 & 0 & 0 & 1 & 2 & & 1 \\
\hline & & 2 & 0 & 0 & 2 & 1 & & 2 \\
\hline & & 3 & 0 & 0 & 3 & 0 & & 3 \\
\hline \multirow[t]{6}{*}{8} & 0 & 0 & 0 & 0 & 2 & 2 & 4 & 2 \\
\hline & & 1 & 0 & 1 & 1 & 1 & & 6 \\
\hline & & 2 & 1 & 0 & 1 & 1 & & 10 \\
\hline & & 3 & 0 & 2 & 0 & 0 & & 8 \\
\hline & & 4 & 1 & 1 & 0 & 0 & & 12 \\
\hline & & 5 & 2 & 0 & 0 & 0 & & 13 \\
\hline 9 & 1 & 0 & 0 & 0 & 2 & 2 & 4 & 2 \\
\hline . & $\ldots$ & $\cdots$ & $\ldots$ & $\ldots$ & $\ldots$ & $\ldots$ & $\ldots$ & $\cdots$ \\
\hline
\end{tabular}
$(N, l)$ appears more than once in the last two columns, this means that the Clebsch-Gordan coefficients corresponding to $\left\langle L l_{\rho} l_{\lambda} M_{L} \mid l_{\rho} l_{\lambda} m_{\rho} m_{\lambda}\right\rangle$ are different from 1.

TABLE IV. This defines the one-to-one correspondence between the barred spin index $\bar{S}_{i}$ for the computer code and the three coupled quark spin quantum numbers $\left(S, M_{S}, S_{12}\right)$.

\begin{tabular}{lccc}
\hline \hline $\bar{S}_{i}$ & $S$ & $S_{12}$ & $M_{S}$ \\
\hline 0 & $1 / 2$ & 0 & $-1 / 2$ \\
1 & $1 / 2$ & 0 & $+1 / 2$ \\
2 & $1 / 2$ & 1 & $-1 / 2$ \\
3 & $1 / 2$ & 1 & $+1 / 2$ \\
4 & $3 / 2$ & 1 & $-3 / 2$ \\
5 & $3 / 2$ & 1 & $-1 / 2$ \\
6 & $3 / 2$ & 1 & $+1 / 2$ \\
7 & $3 / 2$ & 1 & $+3 / 2$ \\
\hline \hline
\end{tabular}


The basis functions of fixed $\left(J, M_{J}\right)$ are given by

$$
\phi^{\left(J, M_{J}\right), \bar{N}, \bar{l}, \bar{R}_{i}}\left(\mathbf{p}_{\rho}, \mathbf{p}_{\lambda}, S_{i}\right)=\sum_{m_{\rho}=-l_{\rho}^{\bar{N}, \bar{l}}}^{l_{\rho}^{\bar{N}, \bar{l}}} \sum_{m_{\lambda}=-l_{\lambda}^{\bar{N}, \bar{l}}}^{l_{\lambda}^{\bar{N}, \bar{l}}} \sum_{R_{i}=0}^{7} \boldsymbol{\phi}_{\left[m_{\rho}\right]\left[m_{\lambda}\right]\left[R_{i}\right]}^{\left.\left(J, M_{J}\right)[\bar{N}] \bar{l}\right]\left[\bar{R}_{i}\right]} \mathcal{B}_{N^{\bar{N}}, l^{\bar{N}, \bar{l}}, m_{\rho}, m_{\lambda}, R_{i}}^{\alpha}\left(\mathbf{p}_{\rho}, \mathbf{p}_{\lambda}, S_{i}\right),
$$

where the array $\phi$ of coefficients is in turn

$$
\begin{aligned}
\phi_{\left[m_{\rho}\right]\left[m_{\lambda}\right]\left[R_{i}\right]}^{\left(J, M_{i}\right)[\bar{l}]\left[\overline{R_{i}}\right]}= & \sum_{M_{S}=-S^{\bar{R}_{i}}}^{S^{\bar{R}_{i}}} \sum_{M_{L}=-L^{\bar{N}}}^{L^{\bar{N}}} \sum_{M_{S_{12}}=-S_{12}^{\bar{R}_{i}}}^{S_{12}^{\bar{R}_{i}}}\left\langle l_{\rho}^{\bar{N}, \bar{l}} m_{\rho} l_{\lambda}^{\bar{N}, \bar{l}} m_{\lambda} \mid L^{\bar{N}} M_{L}\right\rangle\left\langle L^{\bar{N}} M_{L} S^{\bar{R}_{i}} M_{S} \mid J M_{J}\right\rangle \\
& \times\left\langle\frac{1}{2} s_{1}^{R_{i}} \frac{1}{2} s_{2}^{R_{i}} \mid S_{12}^{\bar{R}_{i}} M_{S_{12}}\right\rangle\left\langle S_{12}^{\bar{R}_{i}} M_{S_{12}} \frac{1}{2} s_{3}^{R_{i}} \mid S^{\bar{R}_{i}} M_{S}\right\rangle .
\end{aligned}
$$

The distinction between upper and lower indices in $\phi$ in Eq. (5.10) is generically useful when handling arrays of such indices related to basis functions. The upper indices $\left(\bar{N}, \bar{l}, \bar{R}_{i}\right)$ are not summed over in order to evaluate a (basis) function; they are tags. Then the lower ones, such as $\left(m_{\rho}, m_{\lambda}, R_{i}\right)$, are summed over.

In the end, these coefficients $\phi$ describe a unitary transformation between the uncoupled and the coupled basis.

\section{Wave function symmetry}

We need to have a symmetrized momentum-spin-isospin wave function since fermion antisymmetrization is taken care of by the color degree of freedom. A way to formulate symmetry is by enforcing that the functions of the basis be eigenfunctions of the exchange operators, $P^{12}, P^{13}, P^{23}$, $P^{123}$, and $P^{132}$.

All of these exchange operators can be written as functions of $P^{12}$ and $P^{23}$,

$$
\begin{aligned}
P^{123} & =P^{23} P^{12}, \\
P^{132} & =P^{12} P^{23}, \\
P^{13} & =P^{12} P^{23} P^{12},
\end{aligned}
$$

so that if a state is an even eigenstate of both $P^{12}$ and $P^{23}$, it is automatically symmetric for any permutation of three quarks (antisymmetric when accounting for color). So, we only need to consider the effect of these two operators on the basis. Our strategy is then to obtain the eigenfunctions of these symmetry operators.

The fixed- $\left(J, M_{J}\right)$ basis defined in Eqs. (5.6) and (5.10) is still unsymmetrized, so the individual functions are not necessarily eigenfunctions of the operators $P_{i j}$ and they are recombined.

But notice that since that is an eigenbasis of the harmonic oscillator, and the $P_{i j}$ commute with that Hamiltonian, as also with the total spin and orbital angular momentum, the symmetrizer does mix certain quantum numbers, and, in particular, only states within the same shell $N$ are combined.

Thus, symmetrization only needs to mix wave functions within a $\mathrm{HO}$ shell of fixed $N=\left(2 n_{\rho}+l_{\rho}+2 n_{\lambda}+l_{\lambda}\right), L$ (and thus $\bar{N}$ that only depends on these two is not mixed either in the symmetrization/orthonormalization process) and $S, J, m_{J}$. The next step in our computer treatment is then to construct orthonormal sets of basis functions for each HO shell $N$, spin $J$, and a certain spin projection $M_{J}$.

In summary, we seek within each shell the eigenfunctions $\Phi$ of $P_{12}$ and $P_{23}$ for which

$$
P_{12} \Phi=P_{23} \Phi=\Phi .
$$

Since $P_{i j}$ are Hermitian, the basis so generated is automatically orthogonal.

The action of the symmetrizers on the spin and flavor indices amounts to a simple exchange. As for the momenta, we need the representation of the permutation group on the Jacobi coordinates. Let

$$
\left(\begin{array}{c}
\mathbf{p}_{\rho}^{\prime} \\
\mathbf{p}_{\lambda}^{\prime}
\end{array}\right)=P_{\sigma}\left(\begin{array}{l}
\mathbf{p}_{\rho} \\
\mathbf{p}_{\lambda}
\end{array}\right)
$$

then

$$
\begin{aligned}
& \mathbb{\square}=\left(\begin{array}{ll}
1 & 0 \\
0 & 1
\end{array}\right) \\
& P^{12}=\left(\begin{array}{cc}
-1 & 0 \\
0 & 1
\end{array}\right) \quad P^{23}=\left(\begin{array}{cc}
1 / 2 & \sqrt{3} / 2 \\
\sqrt{3} / 2 & -1 / 2
\end{array}\right)
\end{aligned}
$$

that have simple interpretations in the $(\rho, \lambda)$-plane as a reflection and a $60^{\circ}$ rotation. Other $P_{\sigma}$ can be constructed from these matrices to complete the permutation group, but this is not necessary.

Now, since our functions $\phi$ are by construction eigenfunctions of $S_{12}$ and $I_{12}$ and $l_{\rho}$ is fixed, they are already eigenstates of $P^{12}$ without further work, 


$$
P^{12} \phi=(-1)^{l_{\rho}+S_{12}+I_{12}} \phi= \pm \phi,
$$

and the only task is to discard those that are antisymmetric, $P^{12} \phi=-\phi$, from further consideration, which requires minimum bookkeeping. This yields a new basis of functions $\phi_{i}{ }^{\prime}$ similar to the initial one but with $P^{12}$ antisymmetric elements filtered out.

In this new basis, we construct the matrix with elements

$$
\Pi_{i j}=\left\langle\phi_{i}{ }^{\prime}\left|P_{23}\right| \phi_{j}{ }^{\prime}\right\rangle
$$

and diagonalize it,

$$
\Pi_{i j} c_{j}^{k}=\lambda^{k} c_{j}^{k}
$$

Only the eigenstates with $\lambda^{k}=1$ are physically relevant; the others should be ignored. The new basis is given by

$$
\Phi_{i}=\sum_{j} c_{j}^{i} \phi_{j}^{\prime}
$$

The matrix elements of $\Pi$ can be analytically calculated with the help of the Moshinsky-Beveren-Ribeiro coefficients, but this last tedious evaluation is detailed in the appendix.

The resulting final basis satisfies all necessary properties (good $J, M_{J}$, symmetry, and orthonormalization) and is represented on the computer in terms of the factorized HOspin wave functions. The coefficients of expansion must be analogous to the fractional parentage coefficients used in nuclear structure calculations, but we have not followed the analogy.

\section{E. Control of the numerical precision}

Because we are aiming at small mass splittings within chiral multiplets, we need to decrease as much as possible the numerical errors in diagonalizing our Hamiltonian.

There are two difficult numerical errors to control. The first is the convergence of the nine-dimensional integrals to compute each matrix element of the potential in Eq. (4.5). The second is the truncation of the wave function basis composed of a three-dimensional harmonic oscillator basis per Jacobi coordinate. Once this is achieved, the Hamiltonian is a matrix of the order of $10^{3} \times 10^{3}$, and its diagonalization is standard.

The complexity of our numerical task, starting from the construction of a wave function basis with the correct symmetry, led us to develop a full $\mathrm{C}++$ project, developing parallel codes for CPU clusters.

In what concerns the numerical integration, though we need up to nine-dimensional integrations, our past experience [1] suggests that Monte Carlo integration is less accurate than we need, since in Ref. [1] we were able to achieve only a precision of $O(100) \mathrm{MeV}$.
Therefore, we have settled for numerical Gaussian integration. We have nine-dimensional momenta integrals, with three radial-like integrals and six angular integrals. For the radial integrals we perform the change of variable for the $q$ coordinate and use Gauss-Chebyshev quadrature; for $p_{\rho}$ and $p_{\lambda}$, we use Gauss-Laguerre integration, all with up to 12 points. For the angular integrals, we group them in three twodimensional solid angle integrals, and use Lebedev integration with up to 74 points. This part was made by translating the code from [76] to modern $\mathrm{C}++$. In total, per matrix element in our variational basis, we have up to 700 million integration points. Thus, we reduce the numerical integration error down to a negligible $O(2) \mathrm{MeV}$.

Once the numerical integration error is under control, the most important source of uncertainty in this computation is the need to truncate at a finite shell number $N$. As discussed below Eq. (5.5), the total number of basis states grows with $N_{\max }^{4}$, and the number of matrix elements of $H$ to be computed with the square of that. Thus, we have limited $N_{\max }=10$ or 11 , depending on $J^{P}$, for most computations with the exception of $J=13 / 2$ where we find it necessary to go up to 12.

No further effort in this direction is possible for us, as the final runs for the present computations, in PC clusters with 48 cores, lasted for six months.

Since we are limited in the maximum number of harmonic oscillator shells $N_{\max }$, we adopt the extrapolation of our results to $N_{\max }$, corresponding in a sense to the extrapolation to the infinite volume limit of lattice QCD. Notice that the space extent of the harmonic oscillator functions scales like $\left\langle r^{2}\right\rangle \propto N_{\max }$; thus, the volume scales like $N_{\max }{ }^{3 / 2}$. Since there is no theoretical reason to believe that there is no term with $1 / N_{\max }$ dependence, and since this dependence is apparently followed by the four highest shells we compute, we have used this last extrapolation for our results to follow.

To quantify the uncertainty of the neglected upper shells we have looked at three errors. The first is the simple difference of the last computed shells, for example, $E\left(N_{\max }=10\right)-E\left(N_{\max }=8\right)$. Second, we extrapolate to $N_{\max } \rightarrow \infty$ by means of $E\left(N_{\max }\right)=E_{\infty}+\frac{\delta}{N_{\max }^{2}}$, and finally by means of $E\left(N_{\max }\right)=E_{\infty}+\frac{\delta}{N_{\max }}$. We have tabulated and inspected all three for comparison.

Figure 4 shows an example with the direct computations for different $N_{\max }$, and both extrapolations, in this case for the $J=3 / 2$ family of $\Delta$ baryons. The agreement is satisfactory, and the remaining difference with the experimental point must be ascribed to the model Hamiltonian.

In comparing with other works, we have noted that our preferred extrapolation to large shell number, $E_{\infty}+\frac{\delta}{N_{\max }}$, converges more slowly than the exponential one proposed for conventional three-body nuclear calculations in [77]. We take the regression error in the fit as the extrapolation uncertainty. 


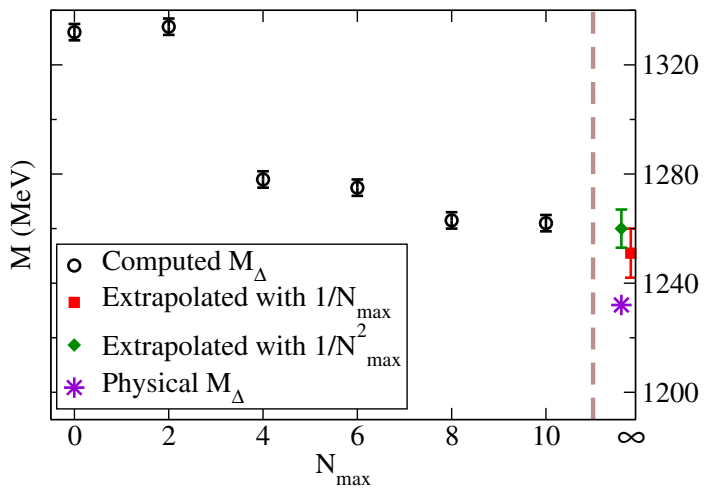

FIG. 4. Black empty circles: computed $M_{\Delta}$ for each $N_{\max }$ maximum shell number as shown, with the error bar representing the numerical integration error. Filled symbols with error bars: extrapolations to $N_{\max }=\infty$. The error bars now include the extrapolation error. Star: physical mass at $1232 \mathrm{MeV}$.

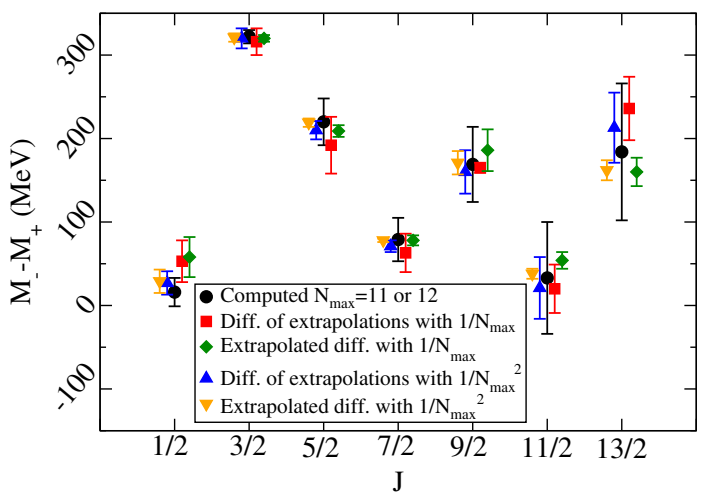

FIG. 5. Comparison of the five methods of estimating the splitting from the variationally truncated Hamiltonian matrix elements, here for the $\Delta$ baryon family.

Proceeding beyond the absolute mass values, we next look at the parity splittings. One can adopt two extrapolation strategies: to take the difference of the extrapolations in the earlier step, or to extrapolate the difference of the computed mass values. While we choose the latter option for subsequent plots, both methods are compared in Fig. 5 and we have tabulated them for all computations as an additional check.

Once we are convinced that we understand the uncertainty in our mass computations, adding the numerical integration error to the extrapolation error, we proceed to report the outcome of the mass splittings in the proposed chiral multiplets.

\section{NUMERICAL RESULTS}

We now proceed to systematically examine our numerical results for the parity splittings $M_{+}-M_{-}$for increasing energy in the baryon spectrum.

We first compare our spectra with the experimental ones. In Fig. 6 we compare our results with the experimental $\Delta^{*}$

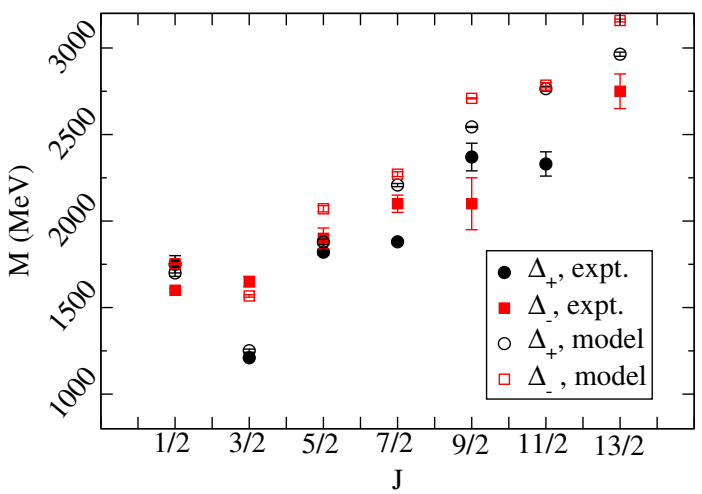

FIG. 6. Comparison of the calculated isospin 3/2 eigenvalues (with positive and negative parity) of the $\Delta$ mass spectrum to the experimental data as collected by the PDG.

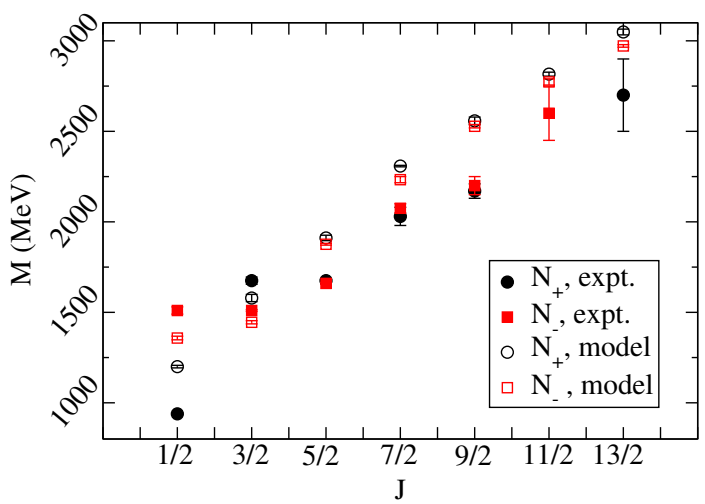

FIG. 7. Same as Fig. 6 but for isospin 1/2 nucleon excitations.

spectrum, and in Fig. 7 we compare our results with the experimental $N^{*}$ spectrum, as a function of the total angular momentum $J$. For clarity, we only show the ground states for each $J$ and parity $P$.

Notice that our model has only one parameter in the interaction, the string tension $\sigma$. In this sense it is comparable to lattice QCD; although it has no dimensional parameter in the Lagrangian, upon quantization a scale appears and lattice QCD gets a dimensional scale, for instance the string tension. Quantitatively, our oneparameter model is not able to get the exact hyperfine splittings of the spectrum, but qualitatively our spectrum, adjusted to have a similar Regge slope, has a good mass splitting between the $\Delta_{+}$and the $\Delta_{-}$and follows the same general trend of the experimental data.

We also illustrate the mass of the first ten radial excitations of a given state, the $\Delta$ with $I=3 / 2$ and $J=$ $3 / 2$ in Fig. 8. In the case of radial excitations, fewer experimental excitations are known, and we only plot the results of our model.

Figures 6-8 illustrate the results we are able to compute in our model. For the first time, we are able to compute the theoretical baryon spectrum in a framework with chiral symmetry, with more states than the experimental data. 


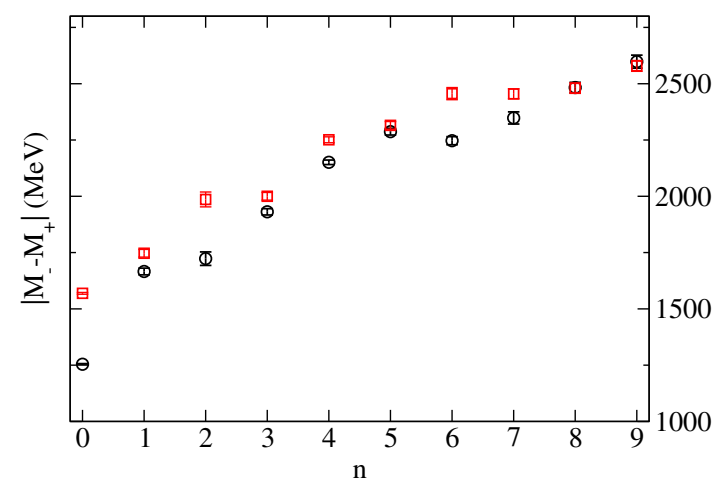

FIG. 8. Mass of the first ten radial excited states for the $I=$ $J=3 / 2 \Delta$ with positive and negative parity, extrapolated from numerical data with up to $N_{\max }=10$.

\section{A. Parity splittings for the $\Delta, I=3 / 2$ case}

We first study in detail the $\Delta, I=3 / 2$ spectrum. This case has the simpler symmetry; because color is antisymmetric and isospin is symmetric, the space $\times$ spin is symmetric.

\section{Splitting of the radial $\Delta$ excitations}

Let us concentrate first on a fixed channel, conveniently chosen to be the $I=J=3 / 2 \Delta$ one.

To assess this, we plot the actual mass of the first ten states with positive parity and also that for the negative parity ones in Fig. 8.

Then one can compare any given state not only with the opposite parity one of the same $n$ but also with the neighboring ones $n \pm 1$, for example. Our results for the parity splittings are shown in Fig. 9. The top plot shows the sequence $\left|M_{+}^{(n)}-M_{-}^{(n)}\right|$ against the excitation number $n$, while the top plot uses the mass of the positive parity partner as the $O X$ axis. There is a visible anticorrelation between the mass of the excited baryon and the parity splitting, but not a discernible power-law fall. But we should not take for granted that, say, the seventh positive parity baryon needs to match the seventh negative parity one (which is what is plotted). It might well be that there is an unequal number of different parity states in the lower spectrum and that they start partnering up only at quite high masses.

There is one way that, within the model calculation, we could enforce the partnering of the states, by employing the quartet states in Eq. (2.8). For example, we could compute the positive parity states up to a given shell and then apply to it the chiral charge $Q_{5}$ as needed to construct its chiral partner. This is man and computing power intensive (because part of the wave function corresponding to the upper shells is out of the truncated variational space) so we have not pursued it further.

Also, it becomes a purely theoretical exercise, as experiment does not have such a handle and is limited to
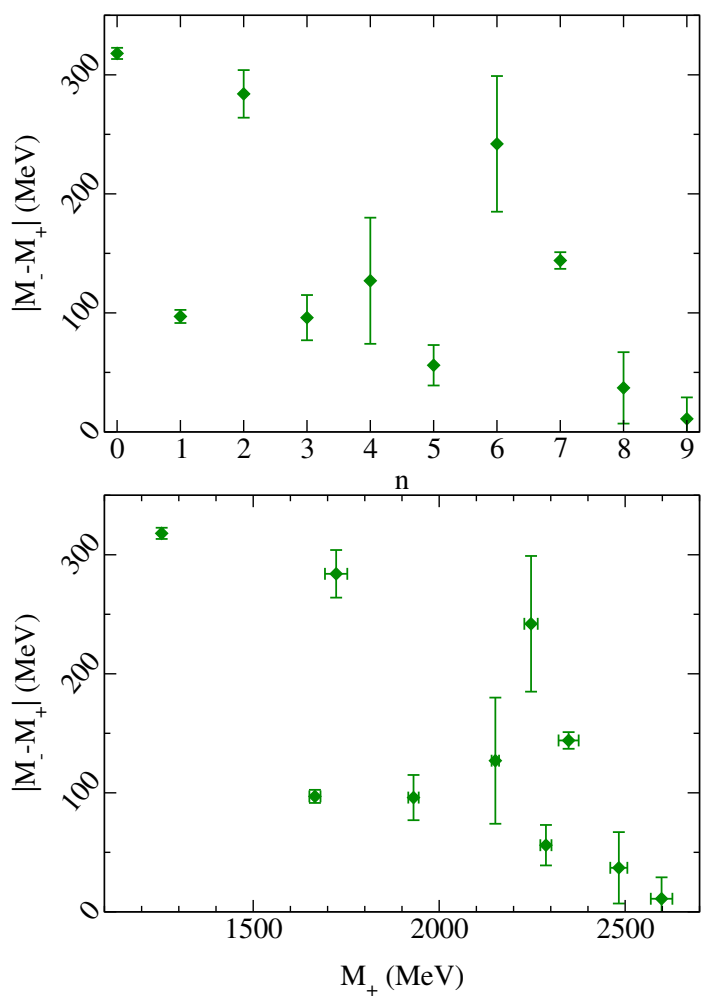

FIG. 9. Mass splittings in the radial excitations of the $I=J=$ $3 / 2 \Delta$ channel. In the $O X$ axis, the top plot shows the number of the excitation and the bottom plot shows the actual mass of the positive parity member of the doublet. In both cases the $O Y$ axis provides the parity mass splittings.

analyzing its results as in Fig. 8, by giving the sequence of increasing masses for a given channel. Moreover, because the experimental states are broad and overlapping, it is unclear whether any excitations beyond the third or fourth are achievable. In all, we find that the most promising alley of experimental investigation is studying increasing angular momentum, and now we turn our attention to it.

\section{Parity splittings in angular $\Delta$ excitations}

We show our results for the splittings for the highly angular excited $\Delta$ baryons in Fig. 10. We remark that the $\Delta$ parity splittings show a decreasing trend for high $J$, but not in a monotonous manner. The decrease is clear for the subspectrum even in $J-3 / 2$, but for the odd $J-3 / 2$ subspectrum we cannot exclude the splitting converging to a finite limit.

Moreover, we compare, in Fig. 11, the model calculation for these $\Delta$ splittings with those obtained with the same code but fixing the quark mass by hand to $M=M(0)=$ constant. This allows one to estimate the effect of the running mass in the spectrum, as discussed in Sec. II B. Moreover, this spoils the Ward identity linking the quark propagator and the pion wave function, and the 


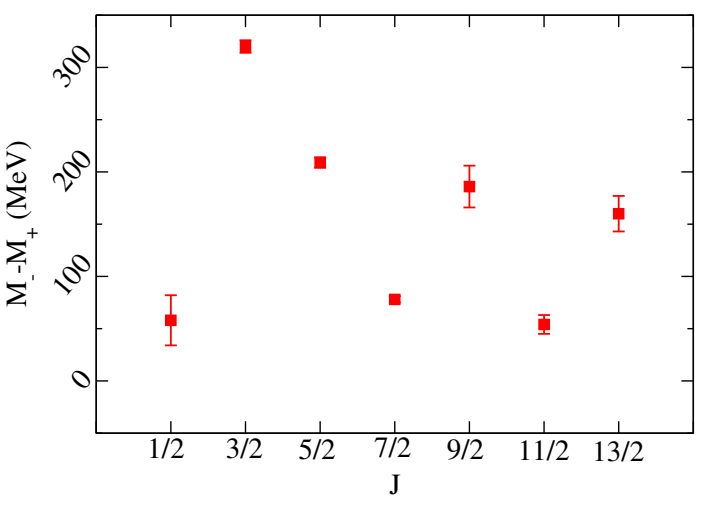

FIG. 10. Parity splittings for $\Delta I=3 / 2$ baryons, as a function of $J$, up to total $J=13 / 2$.

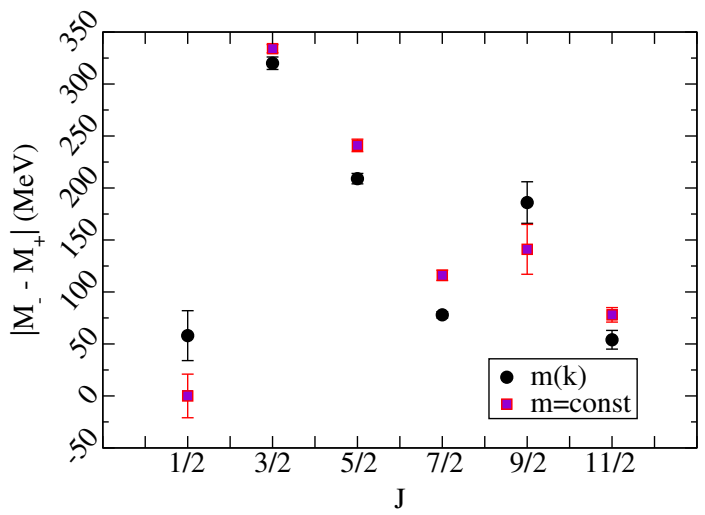

FIG. 11. Comparison of the parity splittings for $\Delta I=3 / 2$ baryons with the running quark mass and with the constant quark mass up to total $J=11 / 2$.

chiral charge is no longer even approximately conserved. In consequence one expects the splittings in this second calculation to lie above those in the chiral computation. Indeed, for $J=3 / 2,5 / 2,7 / 2$, and $11 / 2$ our appreciation is correct and the parity splitting falls faster within the Cornell model with running quark mass than in the constituentlike quark model with fixed mass.

And again, interestingly, for $J=9 / 2$ the a priori expected respective sizes are exchanged.

\section{B. Parity splittings in the possible $\Delta$ Yrast-Yrare quadruplet}

We also study the splittings in the possible chiral quadruplet including the ground state Yrast $n=0$ and the first radial excitation Yrare $n=1$ of the $\Delta I=3 / 2$ as a function of $J$. We compute the splittings up to $J=13 / 2$, as shown in Fig. 12. All states have been computed for different numbers of shells and the splittings extrapolated with $1 / N_{\max }$. In Ref. [6] we discussed the possible subdivision of the quadruplet in two doublets, after diagonalizing Eq. (2.8). Again, this doubling seems to be setting in for $J-3 / 2$ even, not but for $J-3 / 2$ odd.

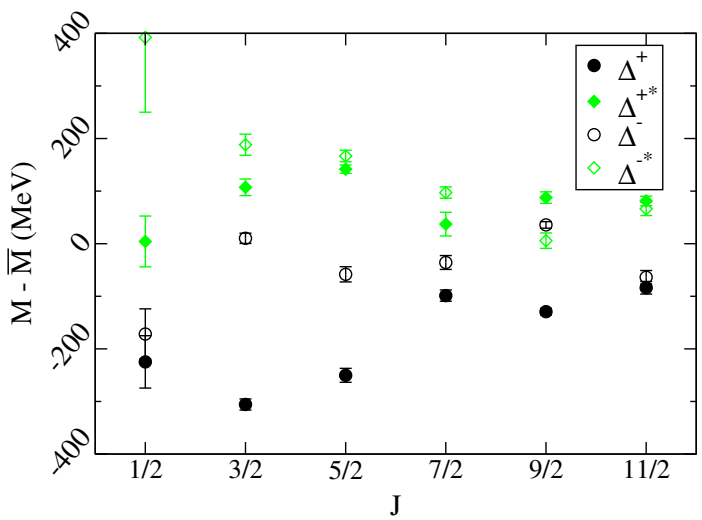

FIG. 12. Parity splittings for the quadruplets including the Yrast and Yrare states with both positive and negative parity as a function of $J$. We show the extrapolation of the ground states and the extrapolation of the first excitations in the $\Delta I=3 / 2$ channel.

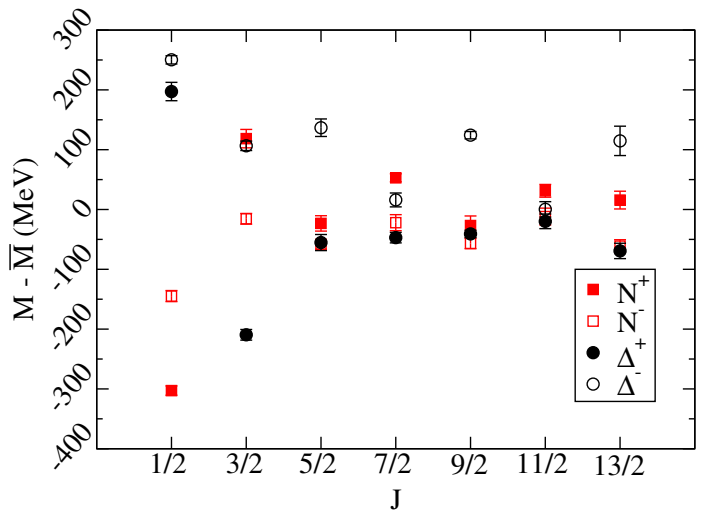

FIG. 13. Yrast nucleon and $\Delta$ parity splittings in the model. The nucleon ones already follow the expected decreasing trend for these moderate (and experimentally accessible to partial wave analysis) angular momenta, while the $\Delta$ splittings still show oscillations and are inconclusive.

\section{Parity splittings in the possible $\Delta-N$ quaduplet}

Figure 13 shows the resulting parity splittings for the quadruplet including both $I=3 / 2 \Delta$ and $I=1 / 2 N$ baryons with $P= \pm 1$, as a function of angular momentum $J$. This is the central outcome of our work.

Again, we observe in our results some level of even-odd staggering. The even $J-3 / 2$ Yrast nucleon (corresponding to $3 / 2,7 / 2$, and $11 / 2$ ) parity splittings decrease very satisfactorily within the quadruplet, and show both the parity and isospin degeneracy expected from insensitivity to chiral symmetry breaking in the high spectrum. However, in the odd $J-3 / 2$ Yrast nucleon (corresponding to $J=1 / 2,5 / 2,9 / 2$, and $13 / 2$ ) the splittings apparently do not converge to 0 for infinitely large $J$.

\section{Quark momentum distributions}

Since the key to dropping parity splittings is in the quark running mass and in the quark momentum distribution, we 


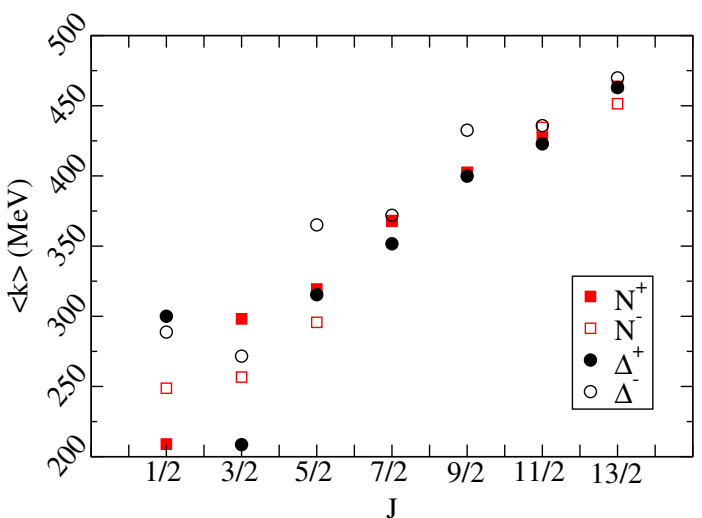

FIG. 14. Average quark momentum $\langle k\rangle$ for each of the positive and negative parity $\mathrm{N}$ and $\Delta$ resonances.

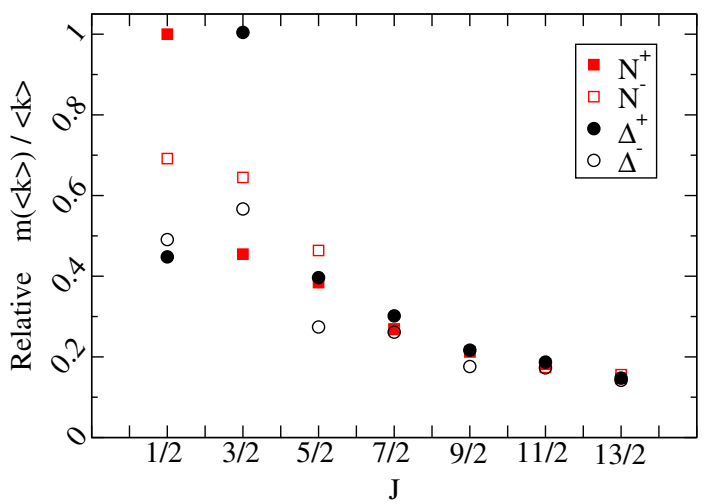

FIG. 15. Drop of the average (running) quark mass across the spectrum due to increasing average momentum. This is fully extracted from the variational computation.

now analyze them in detail. In Fig. 14 we examine the average momentum $\langle k\rangle$ for a quark in each of the states. The average momentum clearly does increase with $J$. This confirms our use of the virial theorem.

Then this has to be compared to the decrease in the quark mass for that average momentum. Figure 15 shows $m(\langle k\rangle) /\langle k\rangle$ for the same states. Since the quark mass in this $\gamma_{0} \gamma_{0}$ model is rather small, we have preferred to divide by the same quantity for the lightest state of each isospinparity combination, so that we only plot the drop relative to that reference point. Clearly, the average of the quark mass decreases with momentum, as we anticipated in our theorem.

Then, for more detail, a different important vista is shown in Fig. 16. The squared wave function in arbitrary units is shown for most of the $\Delta$ states discussed in this work. The top plot displays $|\Psi|^{2}$ in linear scale, the bottom one in logarithmic scale, for best visibility.

It is clearly seen that increasing the angular momentum quantum number $J$ pushes the linear momentum distribution to higher values (towards the right of the plots).
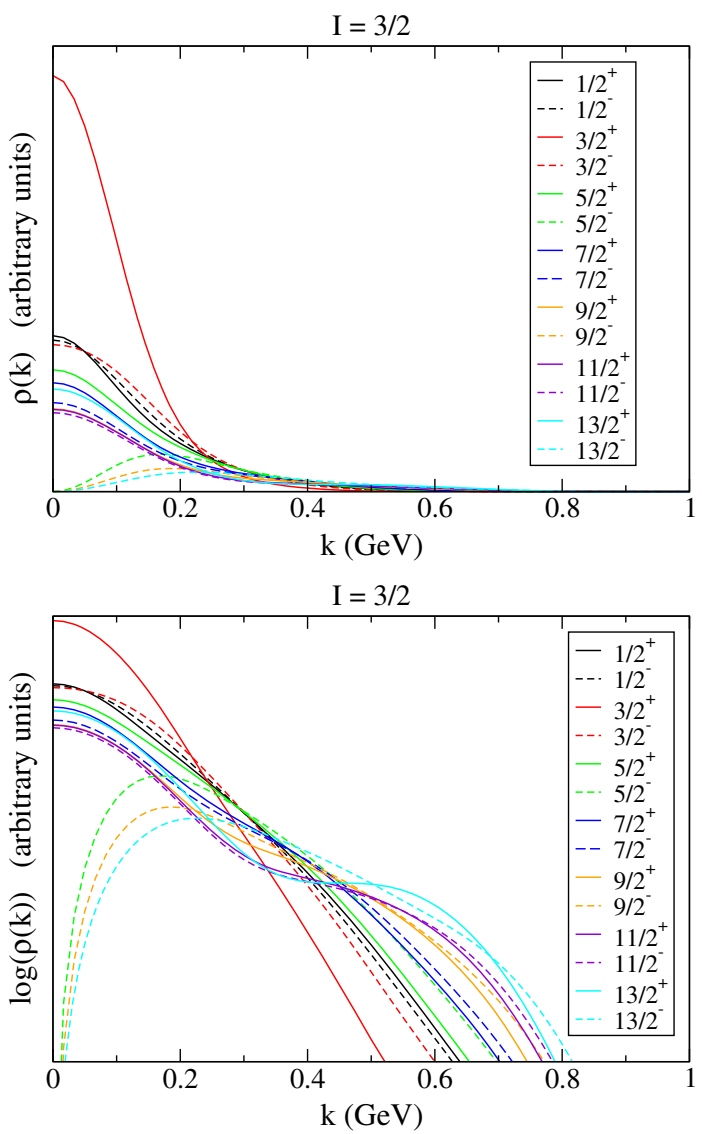

FIG. 16. Momentum wave function squared (top) and its logarithm (bottom) for various $I=3 / 2 \Delta^{J}$ combinations.

There are three states that deserve special mention by their behavior at low momentum. The top line there (corresponding to the ground state $3 / 2^{+}$baryons) is much more peaked at low momentum than the others. But there are three other states, with $J^{P}=5 / 2^{-}, J^{P}=9 / 2^{-}$, and $J^{P}=13 / 2^{-}$, that distinguish themselves by having vanishing wave function for zero average quark momentum.

The reason for this behavior is found examining the wave function basis, and is due to specific quantum number combinatorics. One finds that only in these three cases do we not have a $l_{\lambda}=0$ function on the lowest shell. This means that their splittings to the (differently behaved) positive parity states are larger than otherwise expected, as can be seen in Fig. 11 below. Notice that we would expect, with high $J$, that this component with $l_{\lambda}=0$ and with $\rho(k)=0$ should eventually vanish for all states. Thus, the states $J^{P}=5 / 2^{-}, J^{P}=9 / 2^{-}$, and $J^{P}=13 / 2^{-}$are the ones with the expected behavior.

We can gain even more insight from the further $p_{\rho}-p_{\lambda}$ plane density plots in Fig. 17. For both spins and both parities we see that there are two areas of larger density, one with $\left|p_{\rho}\right|>\left|p_{\lambda}\right|$ and another with $\left|p_{\lambda}\right|>\left|p_{\rho}\right|$. There is a difference, though, distinguishing states with $J-3 / 2$ odd from states with $J-3 / 2$ even. As representative of the 

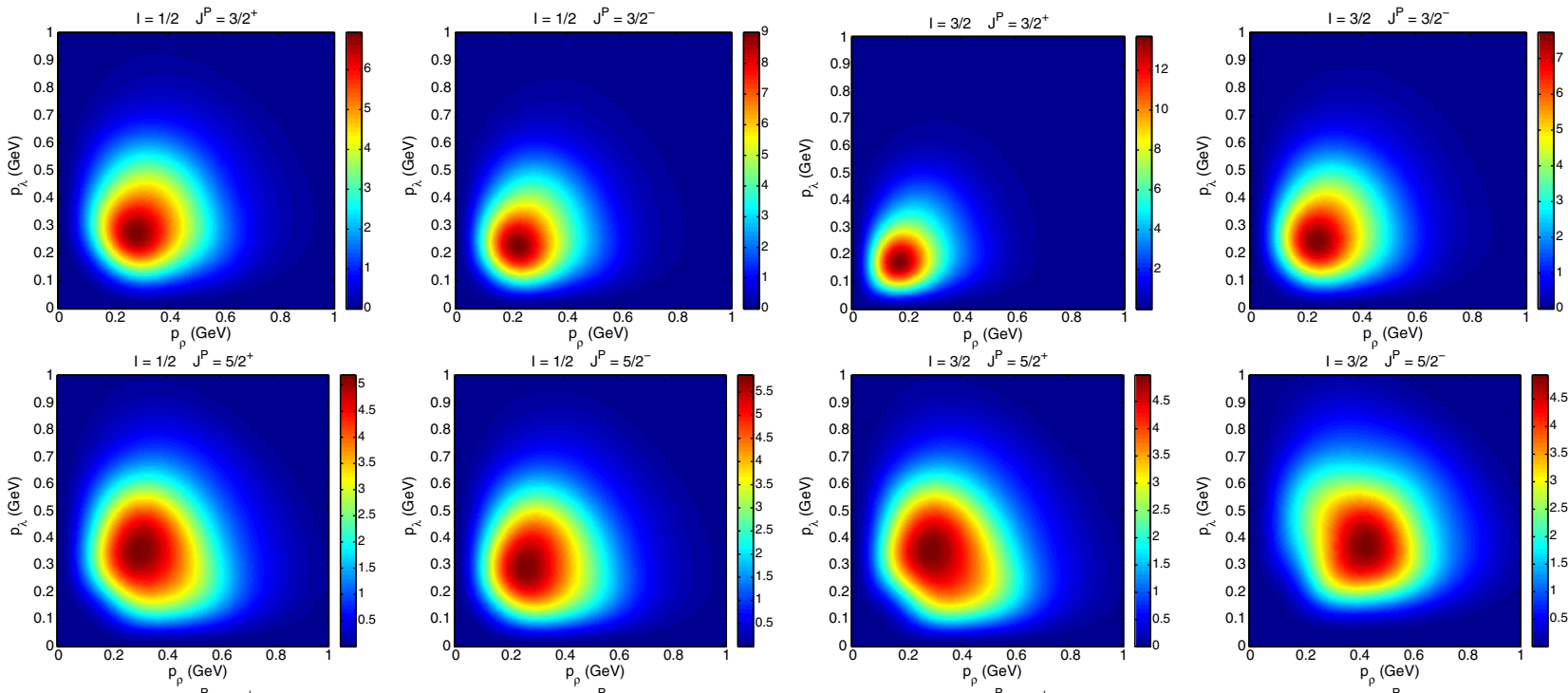

$I=1 / 2 \quad J^{P}=7 / 2^{-}$
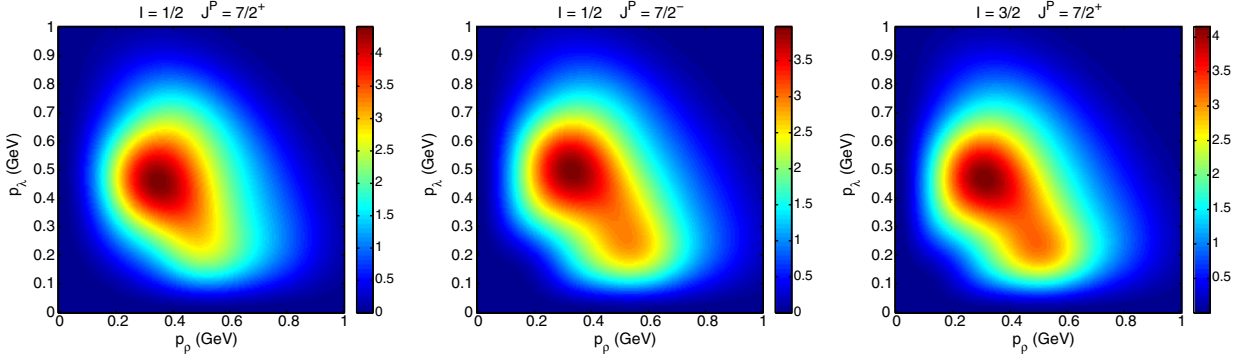

$\mathrm{I}=3 / 2 \quad \mathrm{~J}^{\mathrm{P}}=7 / 2$
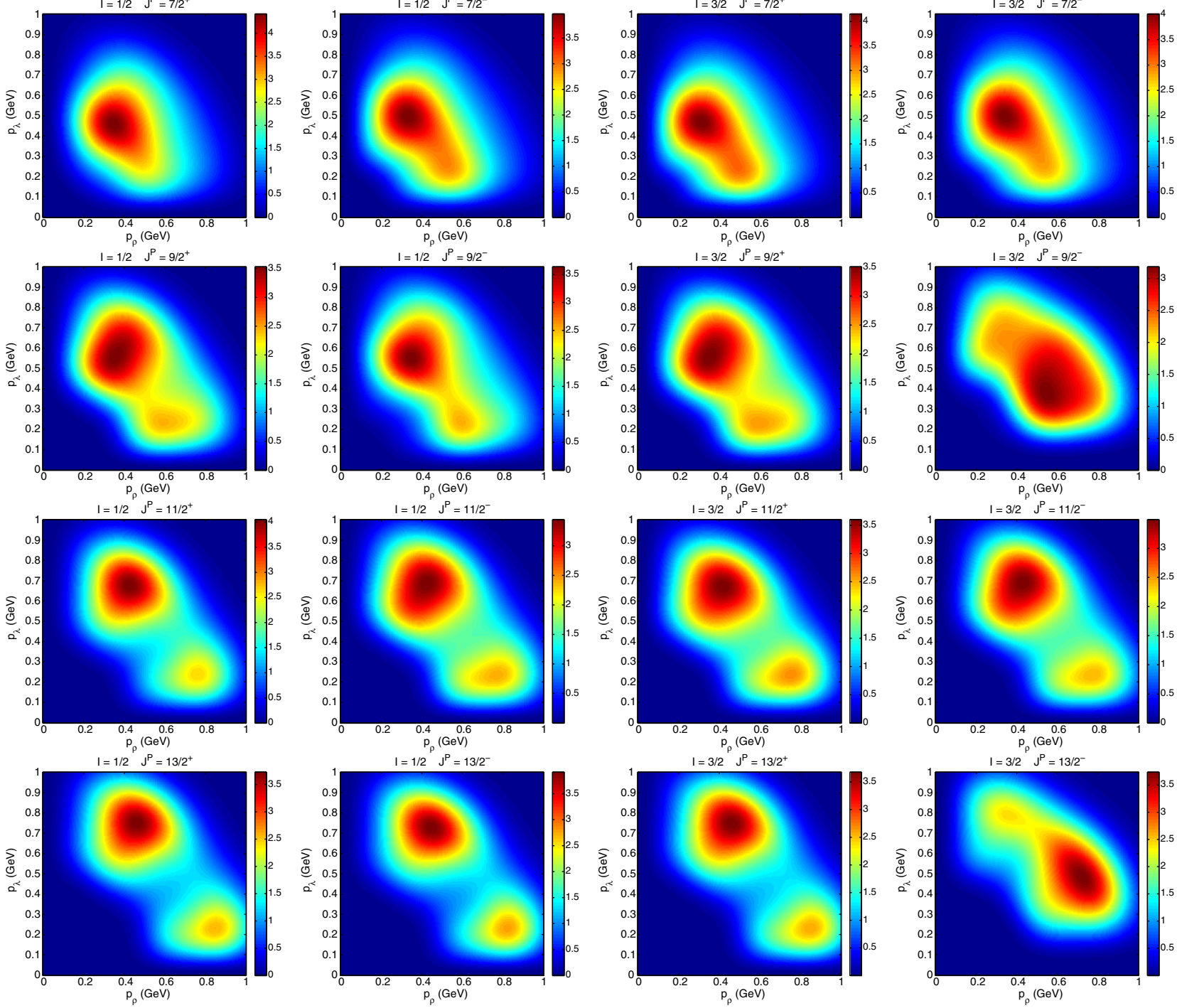

FIG. 17. Momenta density distributions of the Yrast light baryons: from left to right, $N^{+}, N^{-}, \Delta^{+}$, and $\Delta^{-}$and from top to bottom, $J=3 / 2$ to $J=13 / 2$. Starting from $J=7 / 2$, there is a clear general trend as a function of $J$, with the increase of $p_{\lambda}$, except for the $\Delta_{-}$at $J=9 / 2$ and $J=13 / 2$, which follow a different behavior, with the increase of $p_{\rho}$. 
TABLE V. Angular momentum $(L, S)$ composition extracted from the variational computation, in percentage [e.g., 47(2, 3/2) means that $47 \%$ of that state's wave function has $L=2, S=3 / 2$ ]. Fractions smaller than $4 \%$ are omitted.

\begin{tabular}{lcccc}
\hline \hline$J$ & $\left(\frac{1}{2}\right)^{+}$ & $\left(\frac{1}{2}\right)^{-}$ & $\left(\frac{3}{2}\right)^{+}$ & $\left(\frac{3}{2}\right)^{-}$ \\
\hline $1 / 2$ & $100(0,1 / 2)$ & $78(1,3 / 2) 22(1,1 / 2)$ & $97(2,3 / 2)$ & $97(1,1 / 2)$ \\
$3 / 2$ & $42(2,3 / 2) 38(2,1 / 2) 16(1,1 / 2)$ & $69(1,3 / 2) 31(1,1 / 2)$ & $99(0,3 / 2)$ & $97(1,1 / 2)$ \\
$5 / 2$ & $52(2,1 / 2) 47(2,3 / 2)$ & $98(1,3 / 2)$ & $71(2,3 / 2) 29(2,1 / 2)$ & $83(3,3 / 2) 13(2,1 / 2)$ \\
$7 / 2$ & $71(2,3 / 2) 16(4,1 / 2) 10(4,3 / 2)$ & $60(3,3 / 2) 38(3,1 / 2)$ & $98(2,3 / 2)$ & $93(3,1 / 2) 6(3,3 / 2)$ \\
$9 / 2$ & $62(4,1 / 2) 36(4,3 / 2)$ & $97(3,3 / 2)$ & $70(4,3 / 2) 29(4,1 / 2)$ & $72(5,3 / 2) 12(3,3 / 2) 10(4,1 / 2)$ \\
$11 / 2$ & $49(4,3 / 2) 30(6,1 / 2) 18(6,3 / 2)$ & $59(5,3 / 2) 39(5,1 / 2)$ & $98(4,3 / 2)$ & $96(5,1 / 2)$ \\
$13 / 2$ & $64(6,1 / 2) 34(6,3 / 2)$ & $80(5,3 / 2) 9(7,3 / 2) 8(7,1 / 2)$ & $71(6,3 / 2) 29(6,1 / 2)$ & $93(5,3 / 2) 4(7,3 / 2)$ \\
\hline \hline
\end{tabular}

second class of states we have spin $J=11 / 2$ (Fig. 17), and similarly spin $7 / 2$ : the two regions in the $p_{\rho}-p_{\lambda}$ plane are asymmetrically populated, but this asymmetry seems to be equal for both parities. In both it is more likely to have $\left|p_{\lambda}\right|$ larger than $\left|p_{\rho}\right|$. On the contrary, for spin $J=9 / 2$ (Fig. 17) and also for $13 / 2$ we find that the asymmetry in the population of the two regions is not equal for positive and negative parity: in the first case, $\left|p_{\lambda}\right|$ is larger, whereas in the second one, $\left|p_{\rho}\right|$ is larger.

\section{DISCUSSION}

We develop numerical techniques to compute with high precision, for the first time in the framework of chiral invariant quark models, the excited light baryon spectra. We analyze in detail the possible parity doublets both in radial and angular states, both for the $\Delta I=3 / 2$ and nucleon $I=1 / 2$ isospins. While we were not expecting parity doublets in the radial excited spectrum, the setting of parity quadruplets was previously conjectured for high $J$. Notice that for static-light and light-light mesons parity doublets have already been clearly demonstrated in the framework used here.

However, we find an interesting and puzzling result: the spectrum of high $J$ Yrast baryons is not as simple as anticipated, because we have two independent Jacobi coordinates, in momentum space $p_{\rho}$ and $p_{\lambda}$. This is scrutinized in Sec. VID, clearly seen in Fig. 17, where we show the density plots for the momenta density distributions of the Yrast light baryons: from left to right $N^{+}, N^{-}, \Delta^{+}$, and $\Delta^{-}$, and from top to bottom $J=3 / 2$ to $J=13 / 2$. To summarize our analysis of the puzzle

(i) It appears that the wave functions are in general concentrated in the same region of the $\left(p_{\rho}, p_{\lambda}\right)$ plane (with a corresponding approximate degeneracy in the spectrum) except for the $\Delta_{-}$at $J=9 / 2$ and $J=13 / 2$, which follow a different behavior. The general trend, as a function of $J$, is the increase of $p_{\lambda}$, except for the $\Delta_{-}$at $J=9 / 2$ and $J=13 / 2$ where it is $p_{\rho}$ who increases.

(ii) Moreover, most wave functions, except for the $\Delta_{-}$at $J=9 / 2$ and $J=13 / 2$, maintain a small but nonvanishing component at $p \sim 0$.

A further hint in this direction is presented in Table V. There we have extracted the percentage of various RussellSaunders coupling $(L, S)$ wave function for each of the variationally computed states, to a precision of $4 \%$. The effect is clearest for the $\Delta$ baryons that are flavor symmetric, so there is less entanglement. Looking at $\Delta(7 / 2)$ and $\Delta(11 / 2)$ with both parities, we see that the chiral partner wave functions are stepping in $L$ and $S$ : the two percentage distributions are consistent with $(L, S) \rightarrow(L-1, S+1)$ as befits the spin-orbit coupling chiral charge. However, if one focuses instead on $\Delta(9 / 2)$ and $\Delta(13 / 2)$, one sees that there is no simple relation among the wave functions of the would-be parity partners.

Nevertheless, having an exchange in the role of the variables $\left(p_{\rho}, p_{\lambda}\right)$ or having a nonvanishing density at $p \sim$ 0 is, in principle, possible in baryons, even though the wave function in isospin $\times$ spin $\times$ space/momentum is symmetric. Clearly, this is possible only in baryons; it certainly does not occur when there is only one Jacobi coordinate as in mesons. Thus, our unexpected result makes the nucleon and $\Delta$ spectra particularly special within the different hadron spectra.

We now discuss some challenges that should affect any future investigations. First, it is clear that three-quark configurations are only part of the wave function component of excited hadrons, and cannot be trusted through the entire baryon spectrum. Pentaquark correlations are possibly too massive (as no light-quark pentaquark candidate has been shown to be solid), but because Goldstone bosons avoid the mass gap, dynamically generated meson-baryon resonances do seem to play a role in the 
spectrum, with the Roper being a genuine example. If momentum is distributed among more than three quarks, the typical quark momentum is lower than shown in Fig. (16). Thus, chiral symmetry is closer to the Goldstone mode for those states.

It is then questionable whether an even more intensive computational effort than that presented here will be useful to further understand the symmetries of light-quark baryons in generic excitations, unless meson-baryon channels are coupled. That is why we have put the focus on splittings for the lightest states in each angular momentum channel. One expects these Yrast states to have a sizeable $q q q$ component as the short-range chiral interactions (akin to Yukawa potentials) cannot compensate large centrifugal barriers.

Then, it appears interesting to study even higher $J$ to clarify the trends in the spectrum. It will also be interesting to develop other chiral invariant quark models, possibly using Coulomb gauge with a lesser degree of truncation or using a Cornell-like potential [32-34,78], to put model uncertainties in check.

With our results at hand, the baryon spectrum is less promising than the meson spectrum for studying chiral symmetry in the Wigner mode and for extracting the quark mass running in the transition between Goldstone and Wigner modes; theory is needed to guide experiment in the selection of quantum numbers. We propose to look at the sequence of even $J-3 / 2$ of baryon spins $3 / 2,7 / 2,11 / 2$ and give up on $5 / 2,9 / 2,13 / 2$; nevertheless, model dependence remains to be addressed and additional work appears necessary.

A further prediction of the possible Wigner-Weyl realization of chiral symmetry in the high spectrum is that, because the second term in Eq. (2.7) is small, the pion decouples from the baryon, so that processes such as $N^{*} \rightarrow$ $N \pi$ become rarer. However, in view of our mass spectra, indepth study of this phenomenon is also better deferred to future work.

\section{ACKNOWLEDGMENTS}

P. B. and M.C. acknowledge the use of the CPU and GPU servers of PtQCD, supported by CFTP, FCT, and NVIDIA and Grant No. FCT UID/FIS/00777/2013. M. C. is supported by FCT under Contract No. SFRH/BPD/ 73140/2010. The work of F. J. L.E. relied on the Spanish Excellence Network on Hadronic Physics Grant No. FIS2014-57026-REDT, and Grants No. UCM:910309 and No. MINECO:FPA2014-53375-C2-1-P. F. J. L. E. also thanks the Department of Energy's Institute for Nuclear Theory at the University of Washington for its partial support and hospitality. T. V C. work was partially performed at Ghent University with support of the Fund for Scientific Research of Flanders.

\section{APPENDIX: ANALYTICAL COMPUTATION OF EXCHANGE-OPERATOR MATRIX ELEMENTS}

We have relegated to this appendix the analytical evaluation of the $P^{23}$ operator in Eq. (5.15) within states of the unsymmetrized basis. These are given by

$$
\begin{aligned}
\left|\phi_{k}\right\rangle= & \left|I I_{12} J M L S S_{12} n_{\rho} l_{\rho} n_{\lambda} n_{\lambda}\right\rangle \\
= & \left|I I_{12}\right\rangle\left\langle J M \mid L M_{l} S M_{s}\right\rangle\left|S M_{s} S_{12}\right\rangle \\
& \times\left\langle L M_{l} \mid l_{\rho} m_{\rho} l_{\lambda} m_{\lambda}\right\rangle\left|n_{\rho} l_{\rho} m_{\rho}\right\rangle\left|n_{\lambda} l_{\lambda} m_{\lambda}\right\rangle
\end{aligned}
$$

and so the matrix elements are

$$
\begin{aligned}
\left\langle\phi_{k}\left|P^{23}\right| \phi_{k^{\prime}}\right\rangle= & \left\langle I I_{12}\left|P^{23}\right| I I_{12}{ }^{\prime}\right\rangle\left\langle S S_{12}\left|P^{23}\right| S S_{12}{ }^{\prime}\right\rangle \\
& \times\left\langle l_{\rho} m_{\rho} l_{\lambda} m_{\lambda} \mid L M_{l}\right\rangle\left\langle L M_{l} \mid l_{\rho}^{\prime} m_{\rho}^{\prime} l_{\lambda}^{\prime} m_{\lambda}^{\prime}\right\rangle \\
& \times\left\langle n_{\rho} l_{\rho} m_{\rho} n_{\lambda} l_{\lambda} m_{\lambda}\left|P^{23}\right| n_{\rho}^{\prime} l_{\rho}^{\prime} m_{\rho}^{\prime} n_{\lambda}^{\prime} l_{\lambda}^{\prime} m_{\lambda}^{\prime}\right\rangle .
\end{aligned}
$$

\section{Momentum-modulus dependent part}

The effect of exchange operators on the momentum, spin, and flavor parts of the unsymmetrized basis wave functions can be separately calculated because of the factorization in Eq. (A2). The momentum-modulus dependent bra-ket, the last term in Eq. (A2), is a known exchange matrix because of the Gaussian times polynomial structure of the $\mathrm{HO}$ basis,

$\left\langle n_{\rho} l_{\rho} m_{\rho} n_{\lambda} l_{\lambda} m_{\lambda}\left|P^{23}\right| n_{\rho}^{\prime} l_{\rho}^{\prime} m_{\rho}^{\prime} n_{\lambda}^{\prime} l_{\lambda}^{\prime} m_{\lambda}^{\prime}\right\rangle=\mathcal{D}_{\left[l^{\prime}\right]\left[m_{\rho}^{\prime}\right]\left[m_{\lambda}^{\prime}\right][\sigma]}^{[N][l]\left[m_{\rho}\right]\left[m_{\lambda}\right]}$.

Indeed, the $\mathcal{D}$ numbers are the MBRB coefficients for the specific case of transforming a product of two harmonic oscillator wave functions. The coefficients are needed when expressing the product of harmonic oscillator wave functions within a shell $N$, with quantum numbers $\left\{\begin{array}{l}n_{\rho}^{N, l} l_{\rho}^{N, l} m_{\rho} \\ n_{\lambda}^{N, l} l_{\lambda}^{N, l} m_{\lambda}\end{array}\right\}$, and with permuted variables, as a linear combination of products of harmonic oscillator wave functions with quantum numbers $\left\{\begin{array}{l}n_{\rho}^{N, l^{\prime}} l_{\rho}^{N, l^{\prime}} m_{\rho}^{\prime} \\ n_{\lambda}^{N, l^{\prime}} l_{\lambda}^{N, l^{\prime}} m_{\lambda}^{\prime}\end{array}\right\}$ and with the original variables. An explicit expression for the $\mathcal{D}$ coefficients is given by (we drop the index $\mathrm{N}$ for the orbital quantum numbers) 


$$
\begin{aligned}
& \mathcal{D}_{\left[l^{\prime}\right]\left[m_{\rho}^{\prime}\right]\left[m_{\lambda}^{\prime}\right][\sigma]}^{[N][l]\left[m_{\lambda}\right]}=\delta\left(2 n_{\rho}^{l}+l_{\rho}^{l}+2 n_{\lambda}^{l}+l_{\lambda}^{l}, 2 n_{\rho}^{l^{\prime}}+l_{\rho}^{l^{\prime}}+2 n_{\lambda}^{l^{\prime}}+l_{\lambda}^{l^{\prime}}\right) \frac{\pi}{4}(-1)^{n_{\rho}^{l^{\prime}}+n_{\lambda}^{l^{\prime}}+n_{\rho}^{l}+n_{\lambda}^{l}} \\
& \times \sqrt{\frac{n_{\rho}^{l^{\prime}} ! n_{\lambda}^{l^{\prime}} ! n_{\rho}^{l} ! n_{\lambda}^{l} ! \Gamma\left(n_{\rho}^{l^{\prime}}+l_{\rho}^{l^{\prime}}+3 / 2\right) \Gamma\left(n_{\lambda}^{l^{\prime}}+l_{\lambda}^{l^{\prime}}+3 / 2\right) \Gamma\left(n_{\rho}^{l}+l_{\rho}^{l}+3 / 2\right) \Gamma\left(n_{\lambda}^{l}+l_{\lambda}^{l}+3 / 2\right)}{\left(2 l_{\rho}^{l^{\prime}}+1\right)\left(2 l_{\lambda}^{l^{\prime}}+1\right)\left(2 l_{\rho}^{l}+1\right)\left(2 l_{\lambda}^{l}+1\right)}} \\
& \times \sum_{n_{\rho \rho}} \sum_{n_{\rho \lambda}} \sum_{n_{\lambda \rho}} \sum_{n_{\lambda \lambda}} \sum_{l_{\rho \rho}} \sum_{m_{\rho \rho}=-l_{\rho \rho}}^{l_{\rho \rho}} \sum_{m_{\rho \lambda}=-l_{\rho \lambda}}^{l_{\rho \lambda}} \sum_{m_{\lambda \rho}=-l_{\lambda \rho}}^{l_{\lambda \rho}} \sum_{m_{\lambda \lambda}=-l_{\lambda \lambda}}^{l_{\lambda \lambda}}\left\langle l_{\rho \rho} m_{\rho \rho} l_{\rho \lambda} m_{\rho \lambda} \mid l_{\rho}^{l^{\prime}} m_{\rho}^{\prime}\right\rangle\left\langle l_{\rho \rho} 0 l_{\rho \lambda} 0 \mid l_{\rho}^{l^{\prime}} 0\right\rangle \\
& \times\left\langle l_{\lambda \rho} m_{\lambda \rho} l_{\lambda \lambda} m_{\lambda \lambda} \mid l_{\lambda}^{\prime} m_{\lambda}^{\prime}\right\rangle\left\langle l_{\lambda \rho} 0 l_{\lambda \lambda} 0 \mid l_{\lambda}^{l^{\prime}} 0\right\rangle\left\langle l_{\rho \rho} m_{\rho \rho} l_{\lambda \rho} m_{\lambda \rho} \mid l_{\rho}^{l} m_{\rho}\right\rangle\left\langle l_{\rho \rho} 0 l_{\lambda \rho} 0 \mid l_{\rho}^{l} 0\right\rangle\left\langle l_{\rho \lambda} m_{\rho \lambda} l_{\lambda \lambda} m_{\lambda \lambda} \mid l_{\lambda}^{l} m_{\lambda}\right\rangle\left\langle l_{\rho \lambda} 0 l_{\lambda \lambda} 0 \mid l_{\lambda}^{l} 0\right\rangle \\
& \times \frac{\left(\mathcal{P}_{\sigma}^{\rho \rho}\right)^{2 n_{\rho \rho}+l_{\rho \rho}}\left(\mathcal{P}_{\sigma}^{\rho \lambda}\right)^{2 n_{\rho \lambda}+l_{\rho \lambda}}\left(\mathcal{P}_{\sigma}^{\lambda \rho}\right)^{2 n_{\lambda \rho}+l_{\lambda \rho}}\left(\mathcal{P}_{\sigma}^{\lambda \lambda}\right)^{2 n_{\lambda \lambda}+l_{\lambda \lambda}}\left(2 l_{\rho \rho}+1\right)\left(2 l_{\rho \lambda}+1\right)\left(2 l_{\lambda \rho}+1\right)\left(2 l_{\lambda \lambda}+1\right)}{n_{\rho \rho} ! n_{\rho \lambda} ! n_{\lambda \rho} ! n_{\lambda \lambda} ! \Gamma\left(n_{\rho \rho}+l_{\rho \rho}+3 / 2\right) \Gamma\left(n_{\rho \lambda}+l_{\rho \lambda}+3 / 2\right) \Gamma\left(n_{\lambda \rho}+l_{\lambda \rho}+3 / 2\right) \Gamma\left(n_{\lambda \lambda}+l_{\lambda \lambda}+3 / 2\right)} .
\end{aligned}
$$

Such an expression deserves quite some explanation. First, the Kronecker $\delta$ ensures that the harmonic oscillator shell number $N$ remains the same and that there is no mixing between $\mathrm{HO}$-wave functions of different $\mathrm{HO}$ shells. The $n$ 's, $l$ 's, and $m$ 's with a single $\rho / \lambda$ subscript are orbital quantum numbers that are not summed over (= external quantum numbers), but are indices to the $\mathcal{D}$ coefficient. The $n$ 's, $l$ 's, and $m$ 's with a double $\rho / \lambda$ subscript are summed over for every $\mathcal{D}$ coefficient. The values that these indices may take depend on the external quantum numbers. More specifically, the following conditions need to be fulfilled:

$$
\begin{aligned}
& 2 n_{\rho \rho}+l_{\rho \rho}+2 n_{\rho \lambda}+l_{\rho \lambda}=2 n_{\rho}^{l^{\prime}}+l_{\rho}^{l^{\prime}}, \\
& 2 n_{\lambda \rho}+l_{\lambda \rho}+2 n_{\lambda \lambda}+l_{\lambda \lambda}=2 n_{\lambda}^{l^{\prime}}+l_{\lambda}^{l^{\prime}}, \\
& 2 n_{\rho \rho}+l_{\rho \rho}+2 n_{\lambda \rho}+l_{\lambda \rho}=2 n_{\rho}^{l}+l_{\rho}^{l}, \\
& 2 n_{\rho \lambda}+l_{\rho \lambda}+2 n_{\lambda \lambda}+l_{\lambda \lambda}=2 n_{\lambda}^{l}+l_{\lambda}^{l} .
\end{aligned}
$$

These conditions give rise to the $\delta$-function in Eq. (A4) and fix the values of $l_{\rho \lambda}, l_{\lambda \rho}$, and $l_{\lambda \lambda}$. Moreover, they also determine the maximum value of the internal $n$-quantum numbers and of $l_{\rho \rho}$,

$$
\begin{aligned}
& n_{\rho \rho}: 0 \rightarrow \operatorname{Min}\left(\frac{2 n_{\rho}^{l^{\prime}}+l_{\rho}^{l^{\prime}}}{2}, \frac{2 n_{\rho}^{l}+l_{\rho}^{l}}{2}\right), \\
& n_{\rho \lambda}: 0 \rightarrow \operatorname{Min}\left(\frac{2 n_{\rho}^{l^{\prime}}+l_{\rho}^{l^{\prime}}}{2}, \frac{2 n_{\lambda}^{l}+l_{\lambda}^{l}}{2}\right), \\
& n_{\lambda \rho}: 0 \rightarrow \operatorname{Min}\left(\frac{2 n_{\lambda}^{l^{\prime}}+l_{\lambda}^{l^{\prime}}}{2}, \frac{2 n_{\rho}^{l}+l_{\rho}^{l}}{2}\right), \\
& n_{\lambda \lambda}: 0 \rightarrow \operatorname{Min}\left(\frac{2 n_{\lambda}^{l^{\prime}}+l_{\lambda}^{l^{\prime}}}{2}, \frac{2 n_{\lambda}^{l}+l_{\lambda}^{l}}{2}\right), \\
& l_{\rho \rho}: 0 \rightarrow \operatorname{Min}\left(2 n_{\rho}^{l^{\prime}}+l_{\rho}^{l^{\prime}}, 2 n_{\rho}^{l}+l_{\rho}^{l}\right),
\end{aligned}
$$

$$
\begin{gathered}
l_{\rho \lambda}=2 n_{\rho}^{l^{\prime}}+l_{\rho}^{l^{\prime}}-2 n_{\rho \rho}-2 n_{\rho \lambda}-l_{\rho \rho}, \\
l_{\lambda \rho}=2 n_{\rho}^{l}+l_{\rho}^{l}-2 n_{\rho \rho}-2 n_{\lambda \rho}-l_{\rho \rho}, \\
l_{\lambda \lambda}=2 n_{\lambda}^{l}+l_{\lambda}^{l}-2 n_{\rho}^{l^{\prime}}-l_{\rho}^{l^{\prime}}+2 n_{\rho \rho}-2 n_{\lambda \lambda}+l_{\rho \rho} .
\end{gathered}
$$

The numbers $\mathcal{P}_{\sigma}^{i j}$ in Eq. (A4) with $i, j=\rho, \lambda$ denote the matrix elements of the permutation matrix with index $\sigma$ in Eq. (5.15), with $\rho \rightarrow 1$ and $\lambda \rightarrow 2$ (e.g., $\mathcal{P}_{\sigma=132}^{\lambda \rho}=\sqrt{3} / 2$ ).

One more remark is in order. Inside the large expression for the MBRB coefficients in Eq. (A4), there are four CG coefficients for which the magnetic quantum numbers are 0 . These four coefficients conspire so that the MBRB coefficients are only nonzero when $\left(l_{\rho}^{l}, l_{\lambda}^{l}\right)$ and $\left(l_{\rho}^{l^{\prime}}, l_{\lambda}^{\prime}\right)$ couple to the same $L[79]$.

In the end, the availability of the analytical result in Eq. (A4) is what motivates our use of the HO basis to variationally expand all wave functions.

\section{Spin-dependent part}

We now turn to the spin terms in the first line of Eq. (A2). The spin state is described by three quantum numbers $S$, $m_{S}$, and $S_{12}$, given in the order $\left|S m_{S} S_{12}\right\rangle$.

If the spin $S$ takes the value $S=3 / 2$, the $S_{12}$ quantum number becomes redundant as it must necessarily be $S_{12}=1$. The action of the exchange operators is also trivial, because the states are completely symmetric in spin and the matrix element needed for Eq. (A2) is given by

$$
\left\langle\frac{3}{2} m_{S} 1\left|P^{23}\right| \frac{3}{2} m_{S}^{\prime} 1\right\rangle=\delta_{m_{S} m_{S}^{\prime}}
$$

For $S=1 / 2$, we can instead have both $S_{12}=0$ and $S_{12}=1$, so for each $m_{S}$ we have two states to consider. We give them for $m_{S}=+1 / 2$,

$$
\left|\frac{1}{2} \frac{1}{2} 0\right\rangle=\frac{|\uparrow \downarrow \uparrow\rangle-|\downarrow \uparrow \uparrow\rangle}{\sqrt{2}}
$$




$$
\left|\frac{1}{2} \frac{1}{2} 1\right\rangle=\frac{2|\uparrow \uparrow \downarrow\rangle-|\uparrow \downarrow \uparrow\rangle-|\downarrow \uparrow \uparrow\rangle}{\sqrt{6}} .
$$

Applying $P^{23}$ to these states results in

$$
P^{23}\left(\begin{array}{l}
\left|\frac{1}{2} \frac{1}{2} 0\right\rangle \\
\left|\frac{1}{2} \frac{1}{2} 1\right\rangle
\end{array}\right)=\left(\begin{array}{cc}
\frac{1}{2} & \frac{\sqrt{3}}{2} \\
\frac{\sqrt{3}}{2} & -\frac{1}{2}
\end{array}\right)\left(\begin{array}{l}
\left|\frac{1}{2} \frac{1}{2} 0\right\rangle \\
\left|\frac{1}{2} \frac{1}{2} 1\right\rangle
\end{array}\right) .
$$

Curiously, this is the same matrix $\mathcal{P}_{23}$ that represents $P^{23}$ in $\rho-\lambda$ momentum space.
As the result is also valid for $m_{S}=-1 / 2$, we can directly write the matrix element for $S=1 / 2$,

$$
\left\langle\frac{1}{2} i m_{S}\left|P^{23}\right| \frac{1}{2} j m_{S}^{\prime}\right\rangle=\mathcal{P}_{23}^{i j} \delta_{m_{S} m_{S}^{\prime}}
$$

where the indices $i, j=1,2$ correspond to $S_{12}, S_{12}^{\prime}=0,1$.

Finally, the computation of the $P^{23}$ exchange matrix elements in isospin space is similar to the one in spin space and we do not repeat the discussion for the sake of brevity.
[1] P. Bicudo, M. Cardoso, T. Van Cauteren, and F. J. LlanesEstrada, Phys. Rev. Lett. 103, 092003 (2009).

[2] F. J. Llanes-Estrada, P. Bicudo, M. Cardoso, and T. Van Cauteren, in Proceedings of the Mini-Workshop Bled 2008: Few-Quark States and the Continuum, Bled, Slovenia, 2008 , p. 61.

[3] F. J. Llanes-Estrada, P. Bicudo, M. Cardoso, and T. Van Cauteren, Acta Phys. Pol. A 2, 313 (2009).

[4] T. Van Cauteren, P. Bicudo, M. Cardoso, and F. J. LlanesEstrada, EPJ Web Conf. 3, 03012 (2010).

[5] F. J. Llanes-Estrada, Proc. Sci. LC2010 (2010) 066.

[6] F. J. Llanes-Estrada and G. M. Navarro, Mod. Phys. Lett. A 27, 1250033 (2012).

[7] C.E. Detar and T. Kunihiro, Phys. Rev. D 39, 2805 (1989).

[8] T. D. Cohen, Rev. Mod. Phys. 68, 599 (1996).

[9] T. D. Cohen and X. D. Ji, Phys. Rev. D 55, 6870 (1997).

[10] L. Y. Glozman, Phys. Lett. B 475, 329 (2000).

[11] D. Jido, T. Hatsuda, and T. Kunihiro, Phys. Rev. Lett. 84, 3252 (2000).

[12] T. D. Cohen and L. Y. Glozman, Phys. Rev. D 65, 016006 (2001).

[13] E. S. Swanson, Phys. Lett. B 582, 167 (2004).

[14] T. D. Cohen and L. Y. Glozman, Mod. Phys. Lett. A 21, 1939 (2006).

[15] L. Y. Glozman, A. V. Nefediev, and J. E. F. T. Ribeiro, Phys. Rev. D 72, 094002 (2005).

[16] R. F. Wagenbrunn and L. Y. Glozman, Phys. Lett. B 643, 98 (2006).

[17] L. Y. Glozman, Phys. Rep. 444, 1 (2007).

[18] P. Bicudo, N. Brambilla, E. Ribeiro, and A. Vairo, Phys. Lett. B 442, 349 (1998).

[19] F. J. Llanes-Estrada and S. R. Cotanch, Phys. Rev. Lett. 84, 1102 (2000).

[20] R. F. Wagenbrunn and L. Y. Glozman, Phys. Rev. D 75, 036007 (2007).

[21] V. K. Sazonov, G. Schaffernak, and R. F. Wagenbrunn, Phys. Rev. D 90, 014004 (2014).

[22] P. Bicudo, arXiv:1512.09191.
[23] A. V. Nefediev, J. E. F. T. Ribeiro, and A. P. Szczepaniak, JETP Lett. 87, 271 (2008).

[24] A. Le Yaouanc, L. Oliver, S. Ono, O. Pene, and J. C. Raynal, Phys. Rev. D 31, 137 (1985).

[25] A. Amer, A. Le Yaouanc, L. Oliver, O. Pene, and J.c. Raynal, Phys. Rev. Lett. 50, 87 (1983).

[26] A. Le Yaouanc, L. Oliver, O. Pene, and J. C. Raynal, Phys. Rev. D 29, 1233 (1984).

[27] A. Le Yaouanc, L. Oliver, O. Pene, and J. C. Raynal, Phys. Lett. 134B, 249 (1984).

[28] A. Amer, A. Le Yaouanc, L. Oliver, O. Pene, and J. C. Raynal, Phys. Rev. D 28, 1530 (1983).

[29] P. J. d. A. Bicudo and J. E. F. T. Ribeiro, Phys. Rev. D 42, 1611 (1990).

[30] P. J. d. A. Bicudo and J. E. F. T. Ribeiro, Phys. Rev. D 42, 1625 (1990).

[31] P. J. d. A. Bicudo and J. E. F. T. Ribeiro, Phys. Rev. D 42, 1635 (1990).

[32] F. J. Llanes-Estrada and S. R. Cotanch, Nucl. Phys. A697, 303 (2002).

[33] J. M. Torres-Rincon and F. J. Llanes-Estrada, Phys. Rev. Lett. 105, 022003 (2010).

[34] F. J. Llanes-Estrada et al., in Proceedings of 3rd International Conference on Nuclear and Particle Physics with CEBAF at Jefferson Lab (NAPP 2010), Dubrovnik, Croatia [Fizika B20, 63 (2011)].

[35] S. Coito, G. Rupp, and E. van Beveren, Phys. Rev. D 84, 094020 (2011).

[36] U. Thoma, in International Workshop XXXIV on Gross Properties of Nuclei and Nuclear Excitations, Hirschegg, 2007 (World Scientific, Singapore, 2006).

[37] A. Wilson et al. (CBELSA/TAPS Collaboration), Phys. Lett. B 749, 407 (2015).

[38] V. Sokhoyan et al. (CBELSA/TAPS Collaboration), Eur. Phys. J. A 51, 95 (2015); 51, 187 (2015).

[39] V. Crede and W. Roberts, Rep. Prog. Phys. 76, 076301 (2013).

[40] D. I. Glazier, Acta Phys. Pol. A 8, 503 (2015).

[41] V. Crede, AIP Conf. Proc. 1701, 020007 (2016). 
[42] A. Anisovich, E. Klempt, A. Sarantsev, and U. Thoma, Eur. Phys. J. A 24, 111 (2005).

[43] A. V. Anisovich and A. V. Sarantsev, Eur. Phys. J. A 30, 427 (2006).

[44] A. V. Anisovich, R. Beck, E. Klempt, V. A. Nikonov, A. V. Sarantsev, and U. Thoma, Eur. Phys. J. A 48, 15 (2012).

[45] I. Denisenko et al., Phys. Lett. B 755, 97 (2016).

[46] U. Loring, K. Kretzschmar, B. C. Metsch, and H. R. Petry, Eur. Phys. J. A 10, 309 (2001).

[47] U. Loring, B. C. Metsch, and H. R. Petry, Eur. Phys. J. A 10, 395 (2001).

[48] U. Loring, B. C. Metsch, and H. R. Petry, Eur. Phys. J. A 10, 447 (2001).

[49] W. Plessas, Int. J. Mod. Phys. A A30, 1530013 (2015).

[50] J. P. Day, K. S. Choi, and W. Plessas, Few-Body Syst. 54, 329 (2013).

[51] L. Theussl, R. F. Wagenbrunn, B. Desplanques, and W. Plessas, Eur. Phys. J. A 12, 91 (2001).

[52] S. L. Adler and A. C. Davis, Nucl. Phys. B244, 469 (1984).

[53] P. Bicudo, J. E. Ribeiro, and J. Rodrigues, Phys. Rev. C 52, 2144 (1995).

[54] K. A. Olive et al. (Particle Data Group Collaboration), Chin. Phys. C 38, 090001 (2014).

[55] R. G. Edwards et al. (Hadron Spectrum Collaboration), Phys. Rev. D 87, 054506 (2013).

[56] J. P. Vary et al., arXiv:1507.04693.

[57] E. Dikmen, A. F. Lisetski, B. R. Barrett, P. Maris, A. M. Shirokov, and J. P. Vary, Phys. Rev. C 91, 064301 (2015).

[58] R. L. Jaffe, D. Pirjol, and A. Scardicchio, Phys. Rev. Lett. 96, 121601 (2006); Phys. Rev. D 74, 057901 (2006).

[59] N. H. Christ and T. D. Lee, Phys. Rev. D 22, 939 (1980); Phys. Scr. 23, 970 (1981).

[60] W. Lucha and F. F. Schoberl, Phys. Rev. Lett. 64, 2733 (1990).

[61] P. J. A. Bicudo, J. E. F. T. Ribeiro, and R. Fernandes, Phys. Rev. C 59, 1107 (1999).
[62] I. C. Cloet, C. D. Roberts, and A. W. Thomas, Phys. Rev. Lett. 111, 101803 (2013).

[63] P. J. A. Bicudo, G. Krein, J.E. F. T. Ribeiro, and J.E. Villate, Phys. Rev. D 45, 1673 (1992).

[64] P. J. A. Bicudo and A. V. Nefediev, Phys. Rev. D 68, 065021 (2003).

[65] P. Bicudo, Nucl. Phys. A870-871, 112 (2011).

[66] K. Langfeld and L. Moyaerts, Phys. Rev. D 70, 074507 (2004); T. Heinzl et al., Phys. Rev. D 78, 034504 (2008).

[67] P. O. Bowman, U.M. Heller, D. B. Leinweber, M. B. Parappilly, A. G. Williams, and J. b. Zhang, Phys. Rev. D 71, 054507 (2005).

[68] F. J. Llanes-Estrada, Report No. UMI-99-92457.

[69] A. P. Szczepaniak and E. S. Swanson, Phys. Rev. D 65, 025012 (2001).

[70] N. Isgur and G. Karl, Phys. Rev. D 19, 2653 (1979); 23, 817 (1981).

[71] M. Moshinsky, Nucl. Phys. 13, 104 (1959).

[72] T. A. Brody, Tables of Transformation Brackets for Nuclear Shell-Model Calculations (Gordon and Breach, New York, 1967).

[73] M. Moshinsky, The Harmonic Oscillator in Modern Physics: From Atoms to Quarks (Gordon and Breach, New York, 1969).

[74] J. E. T. Ribeiro, Z. Phys. C 5, 27 (1980).

[75] E. van Beveren, Z. Phys. C 17, 135 (1983).

[76] V. I. Lebedev and D. N. Laikov, Doklady Mathematics 59, 477 (1999).

[77] R. N. Perez, J. E. Amaro, E. R. Arriola, P. Maris, and J. P. Vary, Phys. Rev. C 92, 064003 (2015).

[78] E. Eichten, K. Gottfried, T. Kinoshita, K. D. Lane, and T.-M. Yan, Phys. Rev. D 17, 3090 (1978).

[79] D. A. Varshalovich, A. N. Moshalev, and V. K. Khersonskii, Quantum Theory of Angular Momentum (World Scientific, Singapore, 1988), specifically Sec. 12.1.5, Eq. (14), p. 456. 